\title{
Incompressible flows with piecewise constant density
}

\author{
Raphaël Danchin ${ }^{1}$ and Piotr Bogusław Mucha ${ }^{2}$ \\ 1. Université Paris-Est, LAMA, UMR 8050 and Institut Universitaire de France, \\ 61 avenue du Général de Gaulle, 94010 Créteil Cedex, France. \\ E-mail: danchin@univ-paris12.fr \\ 2. Instytut Matematyki Stosowanej i Mechaniki, Uniwersytet Warszawski, \\ ul. Banacha 2, 02-097 Warszawa, Poland. \\ E-mail: p.mucha@mimuw.edu.pl
}

\begin{abstract}
We investigate the incompressible Navier-Stokes equations with variable density. The aim is to prove existence and uniqueness results in the case of discontinuous initial density. In dimension $n=2,3$, assuming only that the initial density is bounded and bounded away from zero, and that the initial velocity is smooth enough, we get the local-in-time existence of unique solutions. Uniqueness holds in any dimension and for a wider class of velocity fields. Let us emphasize that all those results are true for piecewise constant densities with arbitrarily large jumps. Global results are established in dimension two if the density is close enough to a positive constant, and in $n$-dimension if, in addition, the initial velocity is small. The Lagrangian formulation for describing the flow plays a key role in the analysis that is proposed in the present paper.
\end{abstract}

MSC: 35Q30, 76D05

Key words: Inhomogeneous Navier-Stokes equations, critical regularity, piecewise constant density, large jumps, Besov spaces, Lagrangian coordinates, discontinuous data.

\section{Introduction}

Incompressible flows are often modeled by the homogeneous Navier-Stokes equations : that is the density of the fluid is assumed to be a constant. However in many applications as blood flows or models of rivers, although the fluid is practically incompressible, the density can not be considered as a constant quantity, as a consequence of the complex structure of the flow due to e.g. a mixture of fluids or pollution (see e.g. [2, 4, 19, 23, 29]). This makes us look at the density as a nonnegative unknown function which has constant values along the stream lines. The simplest model which can capture such a physical property is the so-called inhomogeneous Navier-Stokes system:

$$
\begin{array}{lrr}
\rho_{t}+v \cdot \nabla \rho=0 & \text { in } & \Omega \times(0, T), \\
\rho v_{t}+\rho v \cdot \nabla v-\nu \Delta v+\nabla Q=0 & \text { in } & \Omega \times(0, T), \\
\operatorname{div} v=0 & \text { in } & \Omega \times(0, T), \\
v=0 & \text { on } & \partial \times(0, T), \\
\left.v\right|_{t=0}=v_{0},\left.\quad \rho\right|_{t=0}=\rho_{0} & \text { in } & \Omega .
\end{array}
$$

The unknown functions are: $\rho$ - the density of the fluid, $v$ - its velocity field and $Q$-its pressure. The constant positive viscosity coefficient is denoted by $\nu$. We consider the cases 
where $\Omega$ is a bounded domain of $\mathbb{R}^{n}$, or the whole space $\mathbb{R}^{n}$, and we focus mainly on the physically relevant space dimensions $n=2,3$.

The goal of the present paper is to revisit results concerning the well-posedness issue of (0.1). We concentrate our analysis on the regularity of density. In our recent work [11], we established the existence and uniqueness of solutions to (0.1) in a critical regularity framework which allowed the initial density to be discontinuous. However, a smallness condition over the jumps was required there. In the present work, we want to discard this smallness condition. At the same time, to simplify the presentation, we do not strive for optimal assumptions as concerns the velocity and assume the viscosity coefficient $\nu$ to be constant.

Let us recall (see in particular [13] and the textbooks [3, 20]) that, roughly, from the qualitative viewpoint the classical results for the homogeneous Navier-Stokes equations carry out to (0.1) : on the one hand global (possibly non unique) weak solutions with finite energy may be constructed and on the other hand, if the density is smooth enough, bounded and bounded away from zero, then global-in-time existence and uniqueness results are available in dimension two for arbitrarily large data, and if the velocity is small in dimension three. These latter results require relatively high regularity of the density, though. In particular it has to be at least continuous, and to have some fractional derivatives in suitable Lebesgue spaces (see e.g. [21] or [9]). It is worthwhile to emphasize that for smooth densities one may show the existence of unique solutions even for vacuum states [6] provided the initial data satisfy some compatibility condition. From the viewpoint of applications such results are not so satisfactory: we wish to consider fluids with e.g. piecewise constant densities, a pattern which is of interest to model a mixture of two fluids.

The results of the paper are split into two groups:

- The first group concerns uniqueness and local-in-time existence results in the case where the initial density is just an $L_{\infty}$ positive function bounded away from zero. In particular, one may consider piecewise constant densities with arbitrary large jumps. As regards the existence issue, we have to restrict ourselves to the (physically relevant) dimensions $n=2,3$.

- The second group concerns the global-in-time existence issue. Here we have to make a smallness assumption over the density which, in the case of piecewise constant initial density, implies that the jumps have to be small. Assuming enough smoothness over the velocity, this enables us to prove global existence for (possibly) large velocity if $n=2$, and for small velocity if $n \geq 3$ (an assumption which is also required for the homogeneous Navier-Stokes equations, anyway).

As explained above, in the present paper, we aim at doing minimal assumptions over the density but we do not strive for optimal regularity of the velocity function. As our method relies on estimates for the Stokes system with merely bounded coefficients, the (rather high) regularity of the velocity is somehow prescribed by the technique. An approach to the issue of sharp regularity has been done in [11] in the critical Besov spaces setting. However in [11] we were able to capture discontinuous density with small jumps only. 
The rest of the paper unfolds as follows. The main results are presented in the first section. Then, some preliminary estimates involving the evolutionary Stokes system are proved. Section 3 is devoted to the derivation of System (0.1) in Lagrangian coordinates. In Section 4, we concentrate on the proof of uniqueness results whereas existence results are proved in the last two sections. Technical estimates involving the divergence equation are presented in the Appendix.

\section{Main results}

Let us first recall the basic energy equality for System (0.1) which may be (formally) derived by testing $(0.1)_{2}$ by $v$ :

Lemma 1 Let $(\rho, v)$ be a sufficiently smooth solution to $(0.1)$ over $\Omega \times[0, T]$. Then there holds

$$
\int_{\Omega}\left(\rho|v|^{2}\right)(t, x) d x+2 \nu \int_{0}^{t} \int_{\Omega}|\nabla v(\tau, x)|^{2} d x d \tau=\int_{\Omega}\left(\rho|v|^{2}\right)(0, x) d x \text { for all } t \in[0, T] .
$$

Subsequently if $\rho_{0}$ is positive and bounded away from zero and $v_{0}$ is in $L_{2}(\Omega)$ then we get a control over $v$ in $L_{\infty}\left(0, T ; L_{2}(\Omega)\right)$ and $\nabla v$ in $L_{2}(\Omega \times(0, T))$. Under very rough regularity assumptions (much less than assumed here), the (formal) energy equality (1.1) provides us with an information about low norms of the velocity, which turns out to be crucial for the proof of global results (see in particular the monograph by [20] and the references therein, as regards the proof of global weak solutions with finite energy). Note that (1.1) gives some regularity information over the velocity even for very rough density. We shall see further in the paper a way to get even more regularity information over the velocity without assuming more on the density.

Before listing the main results of the paper, let us introduce a few notation. Concerning the derivatives of functions $f$ depending on both the time variable $t$ and the space variable $x$, we denote by $f_{t}$ the time derivative and by $D f$ the Jacobian matrix of $f$ with respect to the space variable, namely $(D f)_{i, j}=\partial_{j} f^{i}$. The notation $\nabla f$ is reserved for ${ }^{T}(D f)$.

The Lebesgue spaces of measurable functions with integrable $p$-th power is denoted by $L_{p}(\Omega)$. More generally, if $m \in \mathbb{N}$ then $W_{p}^{m}(\Omega)$ denotes the set of $L_{p}(\Omega)$ functions with derivatives of order less than or equal to $m$ in $L_{p}(\Omega)$. Since the Navier-Stokes equations are of parabolic type, it is also natural to introduce parabolic Sobolev spaces $W_{q, p}^{2,1}(\Omega \times(0, T))$ that is the closure of smooth functions for the norm

$$
\|u\|_{W_{q, p}^{2,1}(\Omega \times(0, T))}=\left\|u, \partial_{t} u\right\|_{L_{p}\left(0, T ; L_{q}(\Omega)\right)}+\|u\|_{L_{p}\left(0, T ; W_{p}^{2}(\Omega)\right)} .
$$

Granted with parabolic spaces, one may now define Besov spaces over $\Omega$ as the following trace space:

$$
B_{q, p}^{2-2 / p}(\Omega)=\left\{f: \Omega \rightarrow \mathbb{R} \text { measurable s.t. } f=\left.\bar{f}\right|_{t=0} \text { for some } \bar{f} \in W_{q, p}^{2,1}(\Omega \times(0,1))\right\} .
$$

The norm can be defined from the above definition as a suitable infimum (for more details concerning the Besov spaces we refer to $[5,33])$. 
Our first result states the uniqueness of solutions with merely bounded density, provided the initial velocity is smooth enough.

Theorem 1 Let $n \geq 2$. Assume that $\Omega$ is $\mathbb{R}^{n}$ or a $C^{2}$ bounded domain of $\mathbb{R}^{n}$. Let $\left(\rho^{1}, v^{1}, Q^{1}\right)$ and $\left(\rho^{2}, v^{2}, Q^{2}\right)$ be two solutions to (0.1) with the same initial data, and density bounded and bounded away from 0 . Suppose moreover that for $k=1,2$,

- Case $n=2$ : there exists $q>2$ such that $v^{k} \in W_{q, 2}^{2,1}(\Omega \times(0, T))$ and $\nabla Q^{k} \in$ $L_{2}\left(0, T ; L_{q}(\Omega)\right)$,

- Case $n \geq 3: v^{k} \in W_{n, 2}^{2,1}(\Omega \times(0, T)), \nabla Q^{k} \in L_{2}\left(0, T ; L_{n}(\Omega)\right)$ and, in addition, $\nabla v^{k} \in$ $L_{2}\left(0, T ; L_{\infty}(\Omega)\right)$.

Then $v^{1} \equiv v^{2}, \nabla Q^{1} \equiv \nabla Q^{2}$ and $\rho^{1} \equiv \rho^{2}$.

Remark 1 As regards the inhomogeneous incompressible Navier-Stokes equations, to our knowledge, the "best" uniqueness result with no smallness condition over the density is due to P. Germain in [16]. It does not apply to solutions with piecewise constant densities, though.

The second result complements Theorem 1. It delivers existence of local-in-time regular and unique solutions in dimensions 2 and 3. Again, the initial density just has to be bounded and bounded away from vacuum.

Theorem 2 Let $n=2,3$ and $\Omega$ be a $C^{2}$ bounded domain or be $\mathbb{R}^{n}$. Let $\rho_{0}$ satisfy

$$
m<\rho_{0}<M
$$

for some positive constants, and $v_{0} \in W_{2}^{2}(\Omega)$ be such that $\operatorname{div} v_{0}=0$ and $\left.v_{0}\right|_{\partial \Omega}=0$. Let $n^{*}=2\left(\frac{n+2}{n}\right)$. There exists a unique solution $(\rho, v)$ to System $(0.1)$ on a time interval $[0, T]$ for some $T>0$ such that $\rho(t, \cdot)$ satisfies $(1.4)$ for all $t \in[0, T]$ and

$$
v \in W_{n^{*}, n^{*}}^{2,1}(\Omega \times(0, T)), \quad v_{t} \in L_{\infty}\left(0, T ; L_{2}(\Omega)\right) \quad \text { and } \quad \nabla v_{t} \in L_{2}(\Omega \times(0, T)) .
$$

Remark 2 The critical Sobolev embedding ensures that $W_{2}^{2}(\Omega)$ is continuously embedded in the Besov space $B_{n^{*}, n^{*}}^{2-2 / n^{*}}(\Omega)$. Keeping in mind the definition of this space given in (1.3), the appearance of the parabolic Sobolev space $W_{n^{*}, n^{*}}^{2,1}(\Omega \times(0, T))$ in the above statement does not come up as a surprise. The $W_{2}^{2}(\Omega)$ assumption for $v_{0}$ is needed to ensure that $\left.\left(\partial_{t} v+v \cdot \nabla v\right)\right|_{t=0}$ is in $L_{2}(\Omega)$. At the same time, owing to the low regularity of the density, we do not know how to propagate the $W_{2}^{2}(\Omega)$ regularity for the velocity.

Proofs of Theorems 1 and 2 are based on the analysis of (0.1) in the Lagrangian coordinates defined by the stream lines. Since the density is merely bounded there is an obstacle to apply any bootstrap method in order to improve the regularity of the velocity. The main difficulty is located in the term with the time derivative. To obtain a better information about $v_{t}$, we adopt techniques from the compressible Navier-Stokes system [25, 28] (concerning uniqueness criteria for the compressible Navier-Stokes system in Lagrangian formulation, see also the recent work by D. Hoff). Roughly speaking, we differentiate the 
(Lagrangian) velocity equation once with respect to time, then apply an energy method. This approach via the Lagrangian coordinates requires only $L_{\infty}$ bounds (by above and by below) for the density, provided the velocity has high regularity. That the density is time-independent in the Lagrangian setting, hence is just a given function, is of course fundamental. In comparison, in [9] where the Eulerian framework is used, the initial density has to be in the Besov space $B_{2,1}^{n / 2}\left(\mathbb{R}^{n}\right)$ (which, roughly, means that it has $n / 2$ derivatives in $L_{2}\left(\mathbb{R}^{n}\right)$ ) but the initial velocity therein has only critical regularity, namely it is in $B_{2,1}^{n / 2-1}\left(\mathbb{R}^{n}\right)$ (to be compared with $B_{2,1}^{2}\left(\mathbb{R}^{n}\right)$ and $n=2,3$ here).

To highlight consequences of Theorems 1 and 2, let us consider the case where the initial divergence-free velocity field is in $W_{2}^{2}(\Omega)$ (and vanishes at the boundary), and the initial density $\rho_{0}$ is ${ }^{1}$

$$
\rho_{0}=m+\sigma \chi_{A_{0}},
$$

where $m, \sigma$ are positive constants and $A_{0}$ is a set with a $C^{1}$ boundary. The velocity field $v$ given by Theorem 2 is Lipschitz with respect to the space variable hence generates a unique $C^{1}$ flow $X$ defined by

$$
X(t, y)=y+\int_{0}^{t} v(\tau, X(\tau, y)) d \tau .
$$

Therefore, the density at time $t$ is given by

$$
\rho(t, \cdot)=m+\sigma \chi_{A(t)}, \quad \text { with } A(t):=X\left(t, A_{0}\right) .
$$

As the flow $X$ is at least $C^{1}$, the initial regularity of the boundary of $A(t)$ is preserved and any geometrical catastrophe (e.g. breaking down or self-intersections of the boundary) will not appear : if $A_{0}$ is diffeomorphic to a ball, then $A(t)$ is diffeomorphic to a ball, too. The above case shows that the system (0.1) can model an interaction of two fluids separated by a free interface. Although tracking the regularity of the boundary $\partial A(t)$ is not the main topic of this paper, we see that Theorem 2 ensures that the $C^{1}$ or $C^{1, \alpha}$ regularity (with $\alpha$ small enough) of $\partial A(t)$ is preserved during the evolution. In other words, we have partially solved in an indirect way a complex free boundary problem which has been left as an open question by P.-L. Lions in his book [20]. Let us emphasize that the standard approach for solving problems of such type requires very technical considerations (see e.g. [1, 30, 32]). Furthermore, with our approach, there are no requirements concerning the regularity of the boundary of the set $A_{0}$ : our results hold for any measurable set $A_{0}$.

The above results concern local-in-time analysis. In order to obtain global-in-time solutions, we have to assume that the jumps of the initial density are small enough. The following theorem states that under this sole assumption over the density, and for sufficiently smooth (possibly large) initial velocity fields, global existence holds true.

Theorem 3 Let $\Omega$ be a $C^{2}$ bounded two-dimensional set, or be $\mathbb{R}^{2}$. There exists a constant $c$ depending only on $\Omega$ and such that if $\rho_{0} \in L_{\infty}(\Omega)$ satisfies

$$
\frac{\sup _{x \in \Omega} \rho_{0}(x)-\inf _{x \in \Omega} \rho_{0}(x)}{\inf _{x \in \Omega} \rho_{0}(x)} \leq c
$$

\footnotetext{
${ }^{1}$ Here $\chi_{A_{0}}$ stands for the characteristic function of the set $A_{0}$.
} 
then for all $v_{0} \in B_{4,2}^{1}(\Omega) \cap L_{2}(\Omega)$ with $\operatorname{div} v_{0}=0$ and $\left.v_{0}\right|_{\partial \Omega}=0$, there exists a unique global-in-time solution to System (0.1) such that (1.1) is satisfied and that, for all $T>0$,

$$
v \in W_{4,2}^{2,1}(\Omega \times(0, T)), \quad \nabla Q \in L_{2}\left(0, T ; L_{4}(\Omega)\right) \quad \text { and } \rho \in L_{\infty}(\Omega \times(0, T)) .
$$

In dimension $n \geq 3$, getting global-in-time strong solutions requires also the initial velocity to be small, (an assumption which is needed in the homogeneous case, anyway). Here is our statement:

Theorem 4 Let $\Omega$ be a bounded n-dimensional $C^{2}$ domain. Let $\rho_{0} \in L_{\infty}(\Omega)$ be positive and bounded away from 0 , and $v_{0} \in B_{q, p}^{2-\frac{2}{q}}(\Omega)$ with $1<p<\infty, n<q<\infty$ and $2-2 / p \neq 1 / q$. There exist two constants $c$ and $c^{\prime}$ depending only on $\Omega, p$ and $q$ and such that if

$$
\frac{\sup _{x \in \Omega} \rho_{0}(x)-\inf _{x \in \Omega} \rho_{0}(x)}{\inf _{x \in \Omega} \rho_{0}(x)}<c \text { and }\left\|v_{0}\right\|_{B_{q, p}^{2-\frac{2}{p}}(\Omega)} \leq c^{\prime} \nu,
$$

then there exists a unique global-in-time solution to the inhomogeneous Navier-Stokes system (0.1) such that

$$
v \in W_{q, p}^{2,1}\left(\Omega \times \mathbb{R}_{+}\right), \quad \nabla Q \in L_{p}\left(\mathbb{R}_{+} ; L_{q}(\Omega)\right) \text { and } \rho \in L_{\infty}\left(\Omega \times \mathbb{R}_{+}\right) .
$$

Furthermore, there exist two positive constants $\alpha$ and $C$ depending only on $\Omega, p, q$ and of the lower and upper bounds for $\rho_{0}$ so that for all $t>0$,

$$
\|v\|_{W_{q, p}^{2,1}(\Omega \times(t, t+1))}+\|\nabla P\|_{L_{p}\left(t, t+1 ; L_{q}(\Omega)\right)} \leq C e^{-\alpha t}\left\|v_{0}\right\|_{B_{q, p}^{2-\frac{2}{p}}(\Omega)} .
$$

Theorems 3 and 4 follow from classical maximal regularity techniques. The smallness conditions (1.7) and (1.9) allow to treat the oscillations of the density as a perturbation that may be put in the right-hand side of the estimates.

At the end we would like to underline that most of our results hold for bounded domains and $\mathbb{R}^{n}$. The case of the whole space is easier: there is no boundary condition and solving the divergence equation is simpler, too. One exception is Theorem 4 where the boundedness of the domain is essential here as it provides exponential decay of the energy norm (the whole space case is tractable under stronger conditions over the density, see our recent work in [11]).

\section{Some linear estimates}

A fundamental role will be played by the Stokes system, that is the following linearization of the velocity equation in (0.1):

$$
\begin{array}{lrr}
m u_{t}-\nu \Delta u+\nabla Q=f & \text { in } & \Omega \times(0, T), \\
\operatorname{div} u=\operatorname{div} R & \text { in } & \Omega \times(0, T), \\
u=0 & \text { in } & \partial \Omega \times(0, T), \\
\left.u\right|_{t=0}=u_{0} & \text { in } & \Omega,
\end{array}
$$

where $m$ and $\nu$ are positive constants. 
We shall make an extensive use of the following solvability result for the Stokes system in the $L_{p}\left(0, T ; L_{q}(\Omega)\right)$ framework:

Theorem 5 Let $\Omega$ be a $C^{2}$ bounded subset of $\mathbb{R}^{n}$. Let $1<p, q<\infty, u_{0} \in B_{q, p}^{2-\frac{2}{p}}(\Omega)$, $f \in L_{p}\left(0, T ; L_{q}(\Omega)\right), R \in W_{p}^{1}\left(0, T ; L_{q}(\Omega)\right)$ so that $\operatorname{div} R \in L_{p}\left(0, T ; W_{q}^{1}(\Omega)\right)$. Suppose that

$$
\operatorname{div} u_{0}=\left.\operatorname{div} R\right|_{t=0} \quad \text { and }\left.\quad \vec{n} \cdot R\right|_{\partial \Omega \times(0, T)}=0 .
$$

If $2-2 / p>1 / q$, assume in addition that $u_{0}=0$ at the boundary, otherwise we assume only $u_{0} \cdot \vec{n}=0$ at $\partial \Omega^{2}$. Then there exists a unique solution to $(2.1)$ such that $u \in W_{q, p}^{2,1}(\Omega \times(0, T))$, $\nabla P \in L_{p}\left(0, T ; L_{q}(\Omega)\right)$, and the following estimate is valid:

$$
\begin{aligned}
& \| m u_{t}, \nu \nabla^{2} u, \nabla P\left\|_{L_{p}\left(0, T ; L_{q}(\Omega)\right)}+m^{\frac{1}{p}} \nu^{\frac{1}{p^{\prime}}} \sup _{0 \leq t \leq T}\right\| u(t) \|_{B_{q, p}^{2-\frac{2}{p}}(\Omega)} \\
& \leq C\left(\left\|f, m R_{t}\right\|_{L_{p}\left(0, T ; L_{q}(\Omega)\right)}+\|\nu \operatorname{div} R\|_{L_{p}\left(0, T ; W_{q}^{1}(\Omega)\right)}+m^{\frac{1}{p}} \nu^{\frac{1}{p^{\prime}}}\left\|u_{0}\right\|_{B_{q, p}^{2-\frac{2}{p}}(\Omega)}\right)
\end{aligned}
$$

where $C$ is independent of $m, \nu$ and $T$.

Proof: In the case $R \equiv 0$, this result is classical (see e.g. [17, 24] and the appendix of [7]). The general case follows from this particular case once constructed a suitable vector-field $w: \Omega \times(0, T) \rightarrow \mathbb{R}^{n}$ fulfilling

$$
\operatorname{div} w=\operatorname{div} R \text { in } \Omega, \quad w=0 \quad \text { at } \partial \Omega .
$$

Taking for granted the existence of such a vector-field, the strategy is simple : we set $v=u-w$ and we gather that $v$ has to satisfy

$$
\begin{array}{lrr}
m v_{t}-\nu \Delta v+\nabla Q=f-m w_{t}+\nu \Delta w & \text { in } & \Omega \times(0, T), \\
\operatorname{div} v=0 & \text { in } & \Omega \times(0, T), \\
v=0 & \text { in } & \partial \Omega \times(0, T), \\
\left.v\right|_{t=0}=u_{0}-w_{0} & \text { in } & \Omega .
\end{array}
$$

Therefore, in order to reduce our study to the case $R \equiv 0$, the vector-field $w$ is required to satisfy $w_{t}, D^{2} w \in L_{p}\left(0, T ; L_{q}(\Omega)\right.$ ) (note that this will imply that $w_{0} \in B_{q, p}^{2-2 / p}(\Omega)$, see (1.3)). The fact that such a solution to (2.3) does exist is granted by the following lemma, the proof of which is postponed in Appendix (see Proposition 3):

Lemma 2 Let $R(t, \cdot)$ be a family of vector-fields defined over the $C^{2}$ bounded domain $\Omega$, parameterized by $t \in(0, T)$. Assume that, for some $1<q<\infty$ and $1 \leq p \leq \infty$ we have $\operatorname{div} R \in L_{p}\left(0, T ; W_{q}^{1}(\Omega)\right), R, R_{t} \in L_{q}\left(0, T ; L_{p}(\Omega)\right)$ and $R \cdot \vec{n}=0$ at the boundary.

Then there exists a vector-field $w$ in $L_{p}\left(0, T ; W_{q}^{2}(\Omega)\right)$ vanishing on $\partial \Omega$, fulfilling

$$
\operatorname{div} w=\operatorname{div} R \quad \text { and } \quad \operatorname{div} w_{t}=\operatorname{div} R_{t} \quad \text { in } \Omega
$$

\footnotetext{
${ }^{2}$ For simplicity we exclude the case $2-2 / p=1 / q$.
} 
and the following estimates:

$$
\begin{gathered}
\|w\|_{L_{p}\left(0, T ; W_{q}^{2}(\Omega)\right)} \leq C\|\operatorname{div} R\|_{L_{p}\left(0, T ; W_{q}^{1}(\Omega)\right)}, \\
\left\|w_{t}\right\|_{L_{p}\left(0, T ; L_{q}(\Omega)\right)} \leq C\left\|R_{t}\right\|_{L_{p}\left(0, T ; L_{q}(\Omega)\right)}
\end{gathered}
$$

for some constant $C$ depending only on $q$ and $\Omega$.

Remark 3 The whole space case is easier to deal with for we do not have to take care of boundary conditions (apart from suitable decay at infinity given by the functional setting). Indeed, in order to solve (2.3), one may set

$$
w=-\nabla(-\Delta)^{-1} \operatorname{div} R .
$$

As the corresponding Fourier multiplier is homogenous of degree 0, we readily get (2.4) and (2.5). Therefore, arguing as above and using the standard maximal regularity result for the Stokes system in $\mathbb{R}^{n}$, we conclude to Theorem 5 in the case $\Omega=\mathbb{R}^{n}$ if the Besov space $B_{q, p}^{2-\frac{2}{p}}(\Omega)$ is replaced with the homogeneous Besov space $\dot{B}_{q, p}^{2-\frac{2}{p}}\left(\mathbb{R}^{n}\right)$ and $W_{q}^{k}(\Omega)$, by its homogeneous version $\dot{W}_{q}^{k}\left(\mathbb{R}^{n}\right)$.

Theorem 5 can be viewed as a classical result. In order to prove Theorem 2 we need to adapt it to the case of variable coefficients. Below, we focus on the $L_{2}$ case where only the boundedness of coefficients is needed.

Lemma 3 Let $\Omega$ be a bounded domain of $\mathbb{R}^{n}$, or $\mathbb{R}^{n}$. Let $\eta \in L_{\infty}(\Omega)$ be a time independent positive function, bounded away from zero, and $R$ satisfy the above boundary conditions. Then the solution $(u, \nabla P)$ with $u \in W_{2,2}^{2,1}(\Omega \times(0, T))$ and $\nabla P \in L_{2}(\Omega \times(0, T))$ to the system

$$
\begin{array}{lrr}
\eta u_{t}-\nu \Delta u+\nabla P=f & \text { in } & \Omega \times(0, T), \\
\operatorname{div} u=\operatorname{div} R & \text { in } & \Omega \times(0, T), \\
u=0 & \text { on } & \partial \Omega \times(0, T), \\
\left.u\right|_{t=0}=u_{0} & \text { on } & \Omega,
\end{array}
$$

fulfills

$$
\begin{aligned}
\sqrt{\nu} \sup _{0 \leq t \leq T}\|\nabla u(t)\|_{L_{2}(\Omega)}+\| u_{t}, \nu \nabla^{2} u, \nabla P & \|_{L_{2}(\Omega \times(0, T))} \leq C\left(\left\|f, R_{t}\right\|_{L_{2}(\Omega \times(0, T))}\right. \\
& \left.+\nu\|\operatorname{div} R\|_{L_{2}\left(0, T ; W_{2}^{1}(\Omega)\right)}+\sqrt{\nu}\left\|\nabla u_{0}\right\|_{L_{2}(\Omega)}\right),
\end{aligned}
$$

where $C$ depends on $\inf \eta$ and $\sup \eta$, but is independent of $T$ and $\nu$.

Proof: First we remove the right-hand side of $(2.6)_{2}$ by means of Lemma 2 (or the remark that follows if $\Omega=\mathbb{R}^{n}$ ): we introduce a vector-field $w$ fulfilling (2.3) such that $w \in W_{2,2}^{2,1}(\Omega \times(0, T))$ with the following bound

$$
\left\|\partial_{t} w, \nu D^{2} w\right\|_{L_{2}\left(0, T ; L_{2}(\Omega)\right)} \leq C\left(\|\operatorname{div} R\|_{L_{2}\left(0, T ; W_{2}^{1}(\Omega)\right)}+\left\|R_{t}\right\|_{L_{2}(\Omega \times(0, T))}\right) .
$$

Hence we may reduce the proof to the case $R \equiv 0$. Now, we observe that testing by $u_{t}$ gives:

$$
\int_{\Omega} \eta\left|u_{t}\right|^{2} d x+\frac{\nu}{2} \frac{d}{d t} \int_{\Omega}|\nabla u|^{2} d x=\int_{\Omega} f \cdot u_{t} d x
$$


Therefore, integrating in time yields

$$
\nu\|\nabla u(t)\|_{L_{2}(\Omega)}^{2}+\int_{0}^{t}\left\|\sqrt{\eta} u_{t}\right\|_{L_{2}(\Omega)}^{2} d \tau \leq \nu\left\|\nabla u_{0}\right\|_{L_{2}(\Omega)}^{2}+\int_{0}^{t}\|f / \sqrt{\eta}\|_{L_{2}(\Omega)}^{2} d \tau .
$$

Since $\eta$ is a positive time independent function which is pointwise bounded from below and above, we obtain

$$
\left\|u_{t}\right\|_{L_{2}(\Omega \times(0, T))}+\sup _{0 \leq t \leq T} \sqrt{\nu}\|D u(t)\|_{L_{2}(\Omega)} \leq C\left(\|f\|_{L_{2}(\Omega \times(0, T))}+\sqrt{\nu}\left\|D u_{0}\right\|_{L_{2}(\Omega)}\right) .
$$

In order to estimate $D^{2} u$ and $D P$, we rewrite (2.6) as

$$
\begin{array}{ll}
-\nu \Delta u+\nabla P=f-\eta u_{t} & \text { in } \Omega \times(0, T), \\
\operatorname{div} u=0 & \text { in } \Omega \times(0, T), \\
u=0 & \text { at } \partial \Omega(0, T) .
\end{array}
$$

If $\Omega$ is a $C^{2}$ bounded domain then the solvability of (2.11) in the $L_{2}$ framework is clear (see e.g. [15], Th. 6.1, page 231), thus taking into account bounds (2.8) and (2.10) we get (2.7). Lemma 3 is proved. In the $\mathbb{R}^{n}$ case, one may just notice that $\nabla P=$ $-\nabla(-\Delta)^{-1} \operatorname{div}\left(f-\eta u_{t}\right)$. As $f-u_{t}$ is in $L_{2}(\Omega \times(0, T))$, we still get the result, first for $\nabla P$, and next for $\nabla^{2} u$.

\section{The Lagrangian coordinates}

A fundamental point of our analysis is the use of Lagrangian coordinates. In order to define them we solve the following ordinary differential equation (treating $y$ as a parameter):

$$
\frac{d X(t, y)}{d t}=v(t, X(t, y)),\left.\quad X(t, y)\right|_{t=0}=y .
$$

This leads to the following relation between the Eulerian coordinates $x$ and the Lagrangian coordinates $y$ :

$$
X(t, y)=y+\int_{0}^{t} v(\tau, X(\tau, y)) d \tau
$$

Let us list a few basic properties for the Lagrangian change of variables:

Proposition 1 Suppose that $v \in L_{1}\left(0, T ; W_{\infty}^{1}(\Omega)\right)$ with $\left.v \cdot \vec{n}\right|_{\partial \Omega}=0$. Then the solution to System (3.1) exists on the time interval $[0, T], X(t, \Omega)=\Omega$ for all $t \in[0, T)$, and $D_{y} X \in L_{\infty}\left(0, T ; L_{\infty}(\Omega)\right)$ with in addition

$$
\left\|D_{y} X(t)\right\|_{L_{\infty}(\Omega)} \leq \exp \left(\int_{0}^{t}\left\|D_{x} v\right\|_{L_{\infty}(\Omega)} d \tau\right) .
$$

Furthermore

$$
X(t, y)=y+\int_{0}^{t} u\left(t^{\prime}, y\right) d t^{\prime} \quad \text { with } u(t, y):=v(t, X(t, y))
$$


so that DX satisfies

$$
D_{y} X(t, y)=\mathrm{Id}+\int_{0}^{t} D_{y} u\left(t^{\prime}, y\right) d t^{\prime} .
$$

Let $Y(t, \cdot)$ be the inverse diffeomorphism of $X(t, \cdot)$. Then

$$
D_{x} Y(t, x)=\left(D_{y} X(t, y)\right)^{-1} \text { with } x=X(t, y)
$$

and, if $\int_{0}^{t}\left|D_{y} u(t, y)\right| d t^{\prime} \leq 1 / 2$ then

$$
\left|D_{x} Y(t, x)-\mathrm{Id}\right| \leq 2 \int_{0}^{t}\left|D_{y} u\left(t^{\prime}, y\right)\right| d t^{\prime} .
$$

Finally, if $v \in L_{1}\left(0, T ; W_{\infty}^{2}(\Omega)\right)$ then $D_{y} X \in L_{\infty}\left(0, T ; W_{\infty}^{1}(\Omega)\right)$ and

$$
\left|D_{y}^{2} X(t, y)\right| \leq e^{\int_{0}^{t}\left|D_{x} v\left(t^{\prime}, X\left(t^{\prime}, y\right)\right)\right| d t^{\prime}} \int_{0}^{t}\left|D_{x}^{2} v\left(t^{\prime}, X\left(t^{\prime}, y\right)\right)\right| e^{\int_{0}^{t^{\prime}}\left|D_{x} v\left(t^{\prime \prime}, X\left(t^{\prime \prime}, y\right)\right)\right| d t^{\prime \prime}} d t^{\prime} .
$$

and if $v \in L_{1}\left(0, T ; W_{p}^{s}(\Omega)\right)$ with $s>\frac{n}{p}+1$, then $D_{y} X-\operatorname{Id} \in L_{\infty}\left(0, T ; W_{p}^{s-1}(\Omega)\right)$.

Proof: The existence of $X$ for $(t, y) \in(0, T) \times \Omega$ follows from the standard ODE theory, a consequence of Picard's theorem. Inequalities (3.3) and (3.8) follow from (3.2) by differentiation and Gronwall lemma. The higher regularity stems from the fact that, under our assumptions, $W_{p}^{s-1}$ is an algebra (the reader may refer to the appendix of [11] for the proof of similar results in a slightly different context).

Equation (3.5) follows from (3.4), by differentiation. Then (3.7) comes from (3.6) provided $D_{y} X$ - Id is small enough: indeed, we have

$$
D_{x} Y=\left(\mathrm{Id}+\left(D_{y} X-\mathrm{Id}\right)\right)^{-1}=\sum_{k=0}^{+\infty}(-1)^{k}\left(\int_{0}^{t} D_{y} u\left(t^{\prime}, y\right) d t^{\prime}\right)^{k} .
$$

This yields (3.5).

Let us now derive the Navier-Stokes equations (0.1) in the Lagrangian coordinates: we set

$$
\eta(t, y):=\rho(t, X(t, y)), \quad u(t, y):=v(t, X(t, y)) \text { and } P(t, y):=Q(t, X(t, y)) .
$$

We claim that System (0.1) recasts in

$$
\begin{array}{lrr}
\eta_{t}=0 & \text { in } & \Omega \times(0, T), \\
\eta u_{t}-\nu \Delta_{u} u+\nabla_{u} P=0 & \text { in } \Omega \times(0, T), \\
\operatorname{div}_{u} u=0 & \text { in } \Omega \times(0, T), \\
u=0 & \text { on } \partial \Omega \times(0, T), \\
\left.u\right|_{t=t_{0}}=\left.v\right|_{t=t_{0}},\left.\quad \eta\right|_{t=0}=\left.\rho\right|_{t=t_{0}} & \text { in } & \Omega,
\end{array}
$$

where operators $\Delta_{u}, \nabla_{u}, \operatorname{div}_{u}$ correspond to the original operators $\Delta, \nabla$, div , respectively, after performing the change to the Lagrangian coordinates. Index $u$ underlines the dependence with respect to $u$. Let us also notice that, as $v$ and $u$ vanish at the boundary, we do have $X(t, \Omega)=\Omega$ for all $t$. 
So let us now give a formal derivation of (3.10). First, given the definition of $X$, it is obvious from the chain rule that

$$
\partial_{t} \eta(t, y)=\left(\partial_{t} \rho+v \cdot \nabla \rho\right)(t, x) \quad \text { and } \quad \partial_{t} u(t, y)=\left(\partial_{t} v+v \cdot \nabla v\right)(t, x) \quad \text { with } \quad x:=X(t, y) \text {. }
$$

The chain rule also yields

$$
D_{y} P(t, y)=D_{x} Q(X(t, y)) \cdot D_{y} X(t, y) \quad \text { with }\left(D_{y} X\right)_{i j}:=\partial_{y_{j}} X^{i} .
$$

Hence we have

$$
D_{x} Q(t, x)=D_{y} P(t, y) \cdot A(t, y) \quad \text { with } \quad A(t, y):=\left(D_{y} X(t, y)\right)^{-1}=D_{x} Y(t, x) .
$$

Next, we notice that if the transform $X$ is volume preserving then for any smooth enough vector-field $H$ we have

$$
\operatorname{div}_{x} H(x)=\operatorname{div}_{y}(A \bar{H})(y) \text { with } x=X(y) \text { and } \bar{H}(y)=H(x) .
$$

This stems from the following series of computations which uses the fact that $\operatorname{det} A \equiv 1$ and the change of variable $x=X(y)$ : for any smooth $q$ with compact support, we have

$$
\begin{aligned}
\int q(x) \operatorname{div}_{x} H(x) d x & =-\int D_{x} q(x) \cdot H(x) d x \\
& =-\int D_{y} \bar{q}(y) \cdot A(y) \cdot \bar{H}(y) d y \\
& =\int \bar{q}(y) \operatorname{div}_{y}(A \bar{H})(y) d y
\end{aligned}
$$

Combining (3.12) and (3.13), we thus deduce that, in Lagrangian coordinates operators $\nabla$, div and $\Delta$ become

$$
\nabla_{u}:={ }^{T} A \cdot \nabla_{y}, \quad \operatorname{div}_{u}:=\operatorname{div}(A \cdot) \quad \text { and } \quad \Delta_{u}:=\operatorname{div}_{u} \nabla_{u} .
$$

In consequence, we have the following relations that will be of constant use:

$$
\begin{aligned}
& \left(\nabla-\nabla_{u}\right) P=\left(\operatorname{Id}-{ }^{T} A\right) \nabla P, \\
& \left(\Delta-\Delta_{u}\right) u=\operatorname{div}\left(\left(\operatorname{Id}-A^{T} A\right) \nabla u\right) .
\end{aligned}
$$

Let us finally emphasize that, owing to the chain rule, we have

$$
\operatorname{div}_{y}(A \cdot)=\operatorname{div}_{u}=A: D_{y} .
$$

This algebraic relation will be of fundamental importance in our analysis.

The following statement ensures the full equivalence between (0.1) and (3.10) under the assumptions of our results stated in Section 1.

Proposition 2 Let $1<p, q<\infty$. Let $\rho_{0} \in L_{\infty}(\Omega)$ and $(u, P)$ be a solution to (3.10) such that $u \in W_{q, p}^{2,1}(\Omega \times(0, T)), \nabla P \in L_{p}\left(0, T ; L_{q}(\Omega)\right)$ and

$$
\int_{0}^{T}\|\nabla u\|_{L_{\infty}(\Omega)} d t \leq 1 / 2 .
$$

Then

$$
v(t, x)=u(t, y), \quad Q(t, x)=P(t, y) \quad \text { and } \quad \rho(t, x)=\rho_{0}(y)
$$

with $x=X(t, y)$ given by (3.2) defines a $W_{q, p}^{2,1}$-solution to (0.1). 
Conversely, if $\rho \in L_{\infty}(\Omega \times(0, T))$ and $(v, Q)$ with $v \in W_{q, p}^{2,1}(\Omega \times(0, T)), \nabla v \in$ $L_{1}\left(0, T ; L_{\infty}(\Omega)\right)$, and $\nabla Q \in L_{p}\left(0, T ; L_{q}(\Omega)\right)$ is a solution to $(0.1)$ then

$$
u(t, y)=v(t, X(t, y)), \quad P(t, y)=Q(t, X(t, y)) \quad \text { and } \quad \eta=\left.\rho\right|_{t=0}
$$

defines a $W_{q, p}^{2,1}$-solution to (3.10).

Proof: The proof goes along the lines of the corresponding one in the appendix of [11]. Having $D u$ small enough in $L_{1}\left(0, T ; L_{\infty}(\Omega)\right)$ is of course fundamental.

\section{Proof of Theorem 1 - uniqueness}

In this part we prove the uniqueness of solutions to System (3.10) under the assumptions of Theorem 1. Here $\Omega$ is a $C^{2}$ bounded domain, or the whole space. The proof is a straightforward application of Lemma 3 to the equations in the Lagrangian form. The important fact is that we have

$$
\nabla v^{i} \in L_{1}\left(0, T ; L_{\infty}(\Omega)\right) \quad i=1,2 .
$$

Hence, taking $T$ small enough, one may assume with no loss of generality that

$$
\int_{0}^{T}\left\|\nabla v^{i}\right\|_{L_{\infty}(\Omega)} d t<\frac{1}{2},
$$

so that Propositions 1 and 2 apply. In particular the regularity properties of those solutions in Lagrangian coordinates are the same as those of Theorem 1. Hence it suffices to consider two solutions $\left(u^{1}, P^{1}\right)$ and $\left(u^{2}, P^{2}\right)$ to System (3.10) with the same initial data and satisfying the conditions of Theorem 1 .

Then, denoting $A^{i}:=A\left(u^{i}\right)$ (see (3.12)), $\delta u:=u^{1}-u^{2}$ and so on, we get

$$
\begin{aligned}
& \eta \delta u_{t}-\nu \Delta \delta u+\nabla \delta P=-\nu\left[\left(\Delta-\Delta_{u^{1}}\right) u^{1}-\left(\Delta-\Delta_{u^{2}}\right) u^{2}\right] \\
& +\left[\left(\nabla-\nabla_{u^{1}}\right) P^{1}-\left(\nabla-\nabla_{u^{2}}\right) P^{2}\right] \quad \text { in } \quad \Omega \times(0, T), \\
& \operatorname{div} \delta u=\operatorname{div}\left[\left(\operatorname{Id}-A^{1}\right) u^{1}-\left(\operatorname{Id}-A^{2}\right) u^{2}\right] \quad \text { in } \quad \Omega \times(0, T), \\
& \delta u=0 \\
& \text { at } \partial \Omega \times(0, T) \text {, } \\
& \left.\delta u\right|_{t=0}=0 \\
& \text { in } \\
& \Omega \text {. }
\end{aligned}
$$

Let us underline that the boundary condition on $R$ from Lemma 3 is fulfilled, since by definition $u^{1}$ and $u^{2}$ are zero at the boundary. Therefore, keeping (3.17) in mind, we obtain for some constant $C$ depending only on $\nu, \inf \eta, \sup \eta$ and $\Omega$ the inequality

$$
\|\delta u\|_{L_{\infty}\left(0, T ; W_{2}^{1}(\Omega)\right)}+\left\|\delta u_{t}, \nabla^{2} \delta u, \nabla \delta P\right\|_{L_{2}(\Omega \times(0, T))} \leq C\left(I_{1}+I_{2}+I_{3}+I_{4}\right)
$$

with

$$
\begin{aligned}
I_{1} & :=\left\|\left(\nabla-\nabla_{u^{1}}\right) P^{1}-\left(\nabla-\nabla_{u^{2}}\right) P^{2}\right\|_{L_{2}(\Omega \times(0, T))}, \\
I_{2} & :=\left\|\left(\operatorname{Id}-A^{1}\right): D u^{1}-\left(\operatorname{Id}-A^{2}\right): D u^{2}\right\|_{L_{2}\left(0, T ; W_{2}^{1}(\Omega)\right)}, \\
I_{3} & :=\|\left[\left(\Delta-\Delta_{u^{1}}\right) u^{1}-\left(\Delta-\Delta_{u^{2}}\right) u^{2} \|_{L_{2}(\Omega \times(0, T))},\right. \\
I_{4} & :=\left\|\partial_{t}\left[\left(\operatorname{Id}-A^{1}\right) u^{1}-\left(\operatorname{Id}-A^{2}\right) u^{2}\right]\right\|_{L_{2}(\Omega \times(0, T))} .
\end{aligned}
$$


In the following computations, we shall use repeatedly the fact that $\delta A:=A^{2}-A^{1}$ satisfies

$$
\delta A(t)=\left(\int_{0}^{t} D \delta u d \tau\right) \cdot\left(\sum_{k \geq 1} \sum_{0 \leq j<k} C_{1}^{j} C_{2}^{k-1-j}\right) \quad \text { with } \quad C_{i}(t):=\int_{0}^{t} D u^{i} d \tau .
$$

We concentrate on the case $n \geq 3$. We shall indicate how our arguments have to be modified if $n=2$, at the end of the section.

In order to bound $I_{1}$, we write

$$
I_{1}(t) \leq\left\|\left(A^{1}-A^{2}\right) \nabla P^{1}\right\|_{L_{2}(\Omega \times(0, t))}+\left\|\left(\mathrm{Id}-A^{2}\right) \nabla\left(P^{1}-P^{2}\right)\right\|_{L_{2}(\Omega \times(0, t))} .
$$

It is clear that

$$
\left\|\left(\mathrm{Id}-A^{2}\right) \nabla\left(P^{1}-P^{2}\right)\right\|_{L_{2}(\Omega \times(0, t))} \leq C t^{1 / 2}\|\nabla \delta P\|_{L_{2}(\Omega \times(0, t))}\left\|D u^{2}\right\|_{L_{2}\left(0, t ; L_{\infty}(\Omega)\right)} .
$$

Let us notice that, according to $(3.7),(4.4)$ and to the critical Sobolev embedding of $W_{2}^{1}(\Omega)$ in $L_{2^{*}}(\Omega)$ (that is $1 / 2^{*}+1 / n=1 / 2$ ), we have

$$
\begin{aligned}
\|\delta A\|_{L_{\infty}\left(0, t ; L_{2^{*}}(\Omega)\right)} & \leq C\left\|\int_{0}^{t}|\nabla \delta u| d t^{\prime}\right\|_{L_{\infty}\left(0, t ; L_{2^{*}}(\Omega)\right)} \\
& \leq C t^{1 / 2}\left\|D^{2} \delta u\right\|_{L_{2}(\Omega \times(0, t))}
\end{aligned}
$$

with $C$ depending only on the norm of the two solutions on $[0, T]$. Therefore,

$$
\begin{aligned}
\left\|\left(A^{1}-A^{2}\right) \nabla P^{1}\right\|_{L_{2}(\Omega \times(0, t))} & \leq C\|\delta A\|_{L_{\infty}\left(0, t ; L_{2^{*}}(\Omega)\right)}\left\|\nabla P^{1}\right\|_{L_{2}\left(0, t ; L_{n}(\Omega)\right)} \\
& \leq C t^{1 / 2}\left\|D^{2} \delta u\right\|_{L_{2}(\Omega \times(0, t))}\left\|\nabla P^{1}\right\|_{L_{2}\left(0, t ; L_{n}(\Omega)\right)} .
\end{aligned}
$$

Let us now bound $I_{2}$. Note that it suffices to bound the norm in $L_{2}(\Omega \times(0, T))$ of the gradient of the corresponding term. If $\Omega$ is bounded this is a consequence of the PoincaréWirtinger inequality as div $\delta u$ has 0 average over $\Omega$, and if $\Omega=\mathbb{R}^{n}$ this stems from the fact that only the norm in $\dot{W}_{2}^{1}\left(\mathbb{R}^{n}\right)$ is involved (see Remark 3). Now, we notice that

$$
\left(\mathrm{Id}-A^{1}\right): D u^{1}-\left(\mathrm{Id}-A^{2}\right): D u^{2}=-\delta A: D u^{1}+\left(A^{2}-\mathrm{Id}\right): D \delta u \text {. }
$$

First, using the embedding of $W_{2}^{1}(\Omega)$ in $L_{2^{*}}(\Omega)$, and keeping in mind (4.1) and that

$$
D u^{i} \in L_{2}\left(0, T ; L_{\infty}(\Omega)\right) \text { and } D^{2} u^{i} \in L_{2}\left(0, T ; L_{n}(\Omega)\right) \text { for } i=1,2,
$$

we get for all $t \in[0, T]$,

$$
\begin{aligned}
&\left\|D\left(\delta A: D u^{1}\right)\right\|_{L_{2}(\Omega \times(0, t))} \lesssim\left\|\left|D u^{1}\right| \int_{0}^{\tau}\left|D^{2} \delta u\right| d \tau^{\prime}\right\|_{L_{2}(\Omega \times(0, t))}+\left\|\left|D^{2} u^{1}\right| \int_{0}^{\tau}|D \delta u| d \tau^{\prime}\right\|_{L_{2}(\Omega \times(0, t))} \\
& \lesssim t^{1 / 2}\left(\left\|D^{2} \delta u\right\|_{L_{2}(\Omega \times(0, t))}\left\|D u^{1}\right\|_{L_{2}\left(0, t ; L_{\infty}(\Omega)\right)}\right. \\
&\left.\quad+\|D \delta u\|_{L_{2}\left(0, t ; L_{2^{*}}(\Omega)\right)}\left\|D^{2} u^{1}\right\|_{L_{2}\left(0, t ; L_{n}(\Omega)\right)}\right) \\
& \\
& \lesssim t^{1 / 2}\left\|D^{2} \delta u\right\|_{L_{2}(\Omega \times(0, t))} .
\end{aligned}
$$


Second, we have

$$
\begin{aligned}
\left\|D\left(\left(A^{2}-\mathrm{Id}\right): D \delta u\right)\right\|_{L_{2}(\Omega \times(0, t))} \lesssim & \left.\left\|D A^{2} \otimes D \delta u\right\|_{L_{2}(\Omega \times(0, T))}+\|\left(A^{2}-\mathrm{Id}\right) \otimes D^{2} \delta u\right) \|_{L_{2}(\Omega \times(0, t))} \\
\lesssim & t^{1 / 2}\left(\left\|D^{2} u^{2}\right\|_{L_{2}\left(0, t ; L_{n}(\Omega)\right)}\|D \delta u\|_{L_{2}\left(0, t ; L_{2^{*}}(\Omega)\right)}\right. \\
& \left.+\left\|D u^{2}\right\|_{L_{1}\left(0, t ; L_{\infty}(\Omega)\right)}\left\|D^{2} \delta u\right\|_{L_{2}(\Omega \times(0, t))}\right)
\end{aligned}
$$

So finally for all $t \in[0, T]$,

$$
I_{2}(t) \leq C t^{1 / 2}\left\|\nabla^{2} \delta u\right\|_{L_{2}(\Omega \times(0, t))}
$$

with $C$ depending only the norm of the solutions over $[0, T]$. The term $I_{3}$ may be handled along the same lines. Indeed we have

$$
I_{3}(t)=\left\|\operatorname{div}\left(\left(\mathrm{Id}-A^{1 T} A^{1}\right) \nabla u^{1}-\left(\mathrm{Id}-A^{2 T} A^{2}\right) \nabla u^{2}\right)\right\|_{L_{2}(\Omega \times(0, t))} .
$$

Finally, we examine $I_{4}$. Using again (4.4), we get (with the convention that $D u^{1,2}$ denotes the components of $D u^{1}$ and $\left.D u^{2}\right)$ :

$$
\begin{aligned}
& \left\|\partial_{t}\left[\delta A u^{1}\right]\right\|_{L_{2}(\Omega \times(0, t))} \lesssim\left\|D \delta u u^{1}\right\|_{L_{2}(\Omega \times(0, t))}+\left\|\int_{0}^{\tau}|D \delta u| d \tau^{\prime}\left|D u^{1,2}\right|\left|u^{1}\right|\right\|_{L_{2}(\Omega \times(0, t))} \\
& +\left\|\int_{0}^{\tau}|D \delta u| d \tau^{\prime}\left|u_{t}^{1}\right|\right\|_{L_{2}(\Omega \times(0, t))} \\
& \lesssim\|D \delta u\|_{L_{\infty}\left(0, t ; L_{2}(\Omega)\right)}\left\|u^{1}\right\|_{L_{2}\left(0, t ; L_{\infty}(\Omega)\right)} \\
& +t^{1 / 2}\|D \delta u\|_{L_{2}\left(0, t ; L_{2^{*}}(\Omega)\right)}\left(\left\|u_{t}^{1}\right\|_{L_{2}\left(0, t ; L_{n}(\Omega)\right)}+\left\|\left|u^{1}\left\|D u^{1,2} \mid\right\|_{L_{2}\left(0, t ; L_{n}(\Omega)\right)}\right)\right.\right. \\
& \lesssim t^{1 / 2}\left\|D^{2} \delta u\right\|_{L_{2}(\Omega \times(0, t))}+\varepsilon(t)\|D \delta u\|_{L_{\infty}\left(0, t ; L_{2}(\Omega)\right)},
\end{aligned}
$$

with $\lim _{t \rightarrow 0} \varepsilon(t)=0$ because

$$
\partial_{t} u^{1}, u^{1} \otimes D u^{1} \text { and } u^{2} \otimes D u^{1} \text { are in } L_{2}\left(0, T ; L_{n}(\Omega)\right) .
$$

At the same time, we have, for all $t \in[0, T]$,

$$
\begin{aligned}
\left\|\partial_{t}\left(\left(\operatorname{Id}-A^{2}\right) \delta u\right)\right\|_{L_{2}(\Omega \times(0, t))} & \lesssim\left\|D u^{2} \delta u\right\|_{L_{2}(\Omega \times(0, t))}+\left\|\left(\operatorname{Id}-A^{2}\right) \partial_{t} \delta u\right\|_{L_{2}(\Omega \times(0, t))} \\
& \lesssim\|\delta u\|_{L_{\infty}\left(0, t ; L_{n^{*}}(\Omega)\right)}\left\|D u^{2}\right\|_{L_{2}\left(0, t ; L_{n}(\Omega)\right)} \\
& +\left\|D u^{2}\right\|_{L_{1}\left(0, t ; L_{\infty}(\Omega)\right)}\left\|\partial_{t} \delta u\right\|_{L_{2}(\Omega \times(0, t))}
\end{aligned}
$$

So one may conclude that

$$
I_{4}(t) \leq t^{1 / 2}\left\|D^{2} \delta u\right\|_{L_{2}(\Omega \times(0, t))}+\varepsilon(t)\left(\|D \delta u\|_{L_{\infty}\left(0, t ; L_{2}(\Omega)\right)}+\left\|\partial_{t} \delta u\right\|_{L_{2}(\Omega \times(0, t))}\right) .
$$

So finally in the case $n \geq 3$, putting together all the previous inequalities yields for all $t \in(0, T)$

$$
\begin{array}{r}
\|\delta u\|_{L_{\infty}\left(0, t ; W_{2}^{1}(\Omega)\right)}+\left\|\delta u_{t}, \nabla^{2} \delta u, \nabla \delta P\right\|_{L_{2}(\Omega \times(0, t))} \\
\quad \leq \varepsilon(t)\left(\|\delta u\|_{L_{\infty}\left(0, t ; W_{2}^{1}(\Omega)\right)}+\left\|\delta u_{t}, \nabla^{2} \delta u, \nabla \delta P\right\|_{L_{2}(\Omega \times(0, t))}\right)
\end{array}
$$

for some positive function $\varepsilon$ going to 0 at 0 . Uniqueness follows on a sufficiently small time interval, then on the whole interval $[0, T]$ thanks to a standard connectivity (or bootstrap) argument. 
Let us now explain how the arguments have to be modified in the two-dimensional case. One cannot follow exactly the above approach owing to the failure of the embedding of $W_{2}^{1}(\Omega)$ in $L_{\infty}(\Omega)$. So we have to assume slightly higher regularity, namely $\nabla P^{1}, \nabla P^{2} \in$ $L_{2}\left(0, T ; L_{q}(\Omega)\right)$ with $q>2$, and so on. For instance, setting $m \in(2, \infty)$ such that $1 / m+$ $1 / q=1 / 2$, we may write

$$
\begin{aligned}
\left\|\left(A^{1}-A^{2}\right) \nabla P^{1}\right\|_{L_{2}(\Omega \times(0, t))} & \leq C t^{1 / 2}\|\nabla \delta u\|_{L_{2}\left(0, t ; L_{m}(\Omega)\right)}\left\|\nabla P^{2}\right\|_{L_{2}\left(0, t ; L_{q}(\Omega)\right)} \\
& \leq C t^{1 / 2}\left\|D^{2} \delta u\right\|_{L_{2}(\Omega \times(0, t))}\left\|\nabla P^{2}\right\|_{L_{2}\left(0, t ; L_{q}(\Omega)\right)} .
\end{aligned}
$$

The other terms of (4.3) may be handled similarly. The details are left to the reader. Theorem 1 is thus proved.

Remark 4 Here we would like to explain the reason why we use the $W_{2,2}^{2,1}$ regularity for the velocity to establish uniqueness. Concentrate our attention on $n=3$. A direct $L_{2}$-energy method (i.e. testing (4.2) by $\delta$ ) requires our bounding $\left(\nabla-\nabla_{u^{1}}\right) P^{1}-\left(\nabla-\nabla_{u^{2}}\right) P^{2}$ in $L_{1}\left(0, T ; L_{2}(\Omega)\right)$, hence the following computation:

$$
\begin{aligned}
\left|\int_{0}^{T} \int_{\Omega} \delta A \nabla P^{1} \delta u d x d t\right| & \leq C\|\delta A\|_{L_{\infty}\left(0, T ; L_{2}(\Omega)\right)}\left\|\nabla P^{1}\right\|_{L_{2}\left(0, T ; L_{3}(\Omega)\right)}\|\delta u\|_{L_{2}\left(0, T ; L_{6}(\Omega)\right)} \\
& \leq C T^{1 / 2}\left\|\nabla P^{1}\right\|_{L_{2}\left(0, T ; L_{3}(\Omega)\right)}\|\nabla \delta u\|_{L_{2}(\Omega \times(0, T))} .
\end{aligned}
$$

So we need $\nabla P^{1} \in L_{2}\left(0, T ; L_{3}(\Omega)\right)$ which is naturally related to $u^{1} \in W_{3,2}^{2,1}$. In addition integrating by parts in the left-hand side of the above inequality, we need to keep track of $\nabla^{2} \delta$ as well as of $\nabla \delta P$ in $L_{2}(\Omega \times(0, T))$. Those two terms are out of control if resorting only to the basic energy inequality.

\section{Proof of Theorem 2 - existence}

The uniqueness property of the system is important, but to have the full picture of the well-posedness issue, we now have to show that there exist solutions with merely bounded density for which Theorem 1 applies. With the method that is proposed below, much more regularity is needed for the initial velocity. However the assumption over the initial density stays that same: it just has to be bounded and bounded away from zero.

\subsection{A priori estimates}

We first concentrate on the proof of a priori estimates for a smooth solution $(u, P)$ to (3.10). To simplify the presentation, we consider the case where $\Omega$ is a $C^{2}$ bounded domain of $\mathbb{R}^{n}$. The whole space case may be achieved by similar arguments : this is just a matter of using homogeneous norms $\|\cdot\|_{\dot{W}_{2}^{1}\left(\mathbb{R}^{n}\right)}$ and $\|\cdot\|_{\dot{B}_{n^{*}, n^{*}}^{2-2 / n^{*}}\left(\mathbb{R}^{n}\right)}$ and resorting to Remark 3 .

In order to prove a priori estimates for $(u, P)$, let us assume in addition that $T$ has been chosen so that (say)

$$
\int_{0}^{T}\|\nabla u\|_{L_{\infty}(\Omega)} d t \leq 1 / 2 .
$$


This enables us to go from (0.1) to (3.10) (and conversely). For any (possibly large) initial velocity $v_{0} \in B_{n^{*}, n^{*}}^{2-2 / n^{*}}(\Omega)$, and $\rho_{0} \in L_{\infty}(\Omega)$ bounded away from zero, we want to find a bound for a solution $(u, P)$ given by Theorem 2 . In other words, we want to control the following quantity:

$$
\Xi_{(u, P)}(T):=\left\|u_{t}\right\|_{L_{\infty}\left(0, T ; L_{2}(\Omega)\right)}+\left\|\nabla u_{t}\right\|_{L_{2}(\Omega \times(0, T))}+\left\|u_{t}, \nabla^{2} u, \nabla P\right\|_{L_{n^{*}}(\Omega \times(0, T))},
$$

with $n^{*}=2\left(\frac{n+2}{n}\right)$, if $T$ is small enough.

Let us first notice that, by standard Sobolev embedding

$$
\int_{0}^{T}\|\nabla u\|_{L_{\infty}(\Omega)} d t \leq C T^{1-\frac{1}{n^{*}} \Xi_{(u, P)}}(T)
$$

which guarantees (5.1) for small times.

In order to use Lemma 3 we restate System (3.10) as follows (of course $\eta=\rho_{0}$ and $\left.u_{0}=v_{0}\right)$ :

$$
\begin{array}{lr}
\eta u_{t}-\nu \Delta u+\nabla P=-\nu\left(\Delta-\Delta_{u}\right) u+\left(\nabla-\nabla_{u}\right) P & \text { in } \Omega \times(0, T), \\
\operatorname{div} u=\operatorname{div}((\operatorname{Id}-A) u) & \text { in } \Omega \times(0, T), \\
u=0 & \text { on } \partial \Omega \times(0, T), \\
\left.u\right|_{t=0}=u_{0} & \text { in } r .
\end{array}
$$

Then keeping (3.15), (3.16), (3.17), and Proposition 1 in mind, we get for some constant $C=C(\nu, \Omega)$,

$$
\begin{aligned}
\sup _{0 \leq t \leq T}\|u(t)\|_{W_{2}^{1}(\Omega)} & +\left\|u_{t}, \nabla^{2} u, \nabla P\right\|_{L_{2}(\Omega \times(0, T))} \\
& \leq C\left(\|\mathrm{Id}-A\|_{L_{\infty}(\Omega \times(0, T))}\left\|u_{t}, \nabla^{2} u, \nabla P\right\|_{L_{2}(\Omega \times(0, T))}\right. \\
& \left.+\left\|\nabla A \nabla u, A_{t} u\right\|_{L_{2}(\Omega \times(0, T))}+\left\|u_{0}\right\|_{W_{2}^{1}(\Omega)}\right) .
\end{aligned}
$$

The $W_{2,2}^{2,1}(\Omega \times(0, T))$ regularity of the velocity, coming from (5.5), is not sufficient to control the Lagrangian coordinates, namely the terms containing $A$ in the right-hand side of $(5.5)$, because $\nabla W_{2,2}^{2,1}(\Omega \times(0, T))$ is not embedded in $L_{1}\left(0, T ; L_{\infty}(\Omega)\right)$. Hence, to close the estimates, higher regularity is needed. Differentiating (5.4) once with respect to time is the easiest way to achieve it, because it does not affect the irregular density which is time independent in the Lagrangian setting. We get

$$
\begin{aligned}
& \eta u_{t t}-\nu \Delta u_{t}+\nabla_{u} P_{t}= \\
& -\nu\left(\Delta-\Delta_{u}\right) u_{t}+\nu\left(\Delta_{u}\right)_{t} u-\left(\nabla_{u}\right)_{t} P \quad \text { in } \Omega \times(0, T), \\
& \operatorname{div}_{u} u_{t}=-\operatorname{div} A_{t} u \quad \text { in } \Omega \times(0, T) \text {, } \\
& \begin{array}{ll}
u_{t}=0 & \text { on } \partial \Omega \times(0, T) .
\end{array}
\end{aligned}
$$

At this stage the question of the regularity of $\left.u_{t}\right|_{t=0}$ arises. This information can be found out only from the equations. At time $t=0$ the Eulerian and Lagrangian coordinates coincide (that is $A=\mathrm{Id}$ ), so the regularity of $\left.u_{t}\right|_{t=0}$ is just that of $\left.\eta^{-1}(\nabla P-\nu \Delta u)\right|_{t=0}$. 
However the regularity of $\left.\nabla P\right|_{t=0}$ is unknown, so we rather have to use the fact that differentiating $(5.4)_{2}$ with respect to $t$ implies that

$$
\left.\eta u_{t}\right|_{t=0}+\left.\nabla P\right|_{t=0}=\nu \Delta u_{0},\left.\quad \operatorname{div} u_{t}\right|_{t=0}=-\operatorname{div}\left(\left.A_{t}\right|_{t=0} u_{0}\right) .
$$

Note that $\left.A_{t}\right|_{t=0}$ need not be trivial so in order to bound $\left.u_{t}\right|_{t=0}$ in $L_{2}(\Omega)$, we first have to remove its potential part. For that, we use the Bogovskil operator $\mathcal{B}$ (see Lemma 5) setting

$$
\phi=\mathcal{B}\left[-\operatorname{div}\left(\left.A_{t}\right|_{t=0} u_{0}\right)\right] \quad \text { so that } \quad \operatorname{div} \phi=-\operatorname{div}\left(\left.A_{t}\right|_{t=0} u_{0}\right) \text { in } \Omega, \quad \phi=0 \text { at } \partial \Omega \text {. }
$$

Let us notice that, because

$$
A(t, y) \cdot D X(t, y)=\mathrm{Id} \text { and }\left.D X\right|_{t=0}=\mathrm{Id} \quad \text { with } \quad X(t, y)=\mathrm{Id}+\int_{0}^{t} u(\tau, y) d \tau
$$

we have $\left.A_{t}\right|_{t=0}=-D u_{0}$, hence $\left.A_{t}\right|_{t=0} u_{0}=-\left(u_{0} \cdot \nabla u_{0}\right)=-\operatorname{div}\left(u_{0} \otimes u_{0}\right)$.

Now, $W_{2}^{2}(\Omega)$ is an algebra if $n=2,3$. Hence $\left.A_{t}\right|_{t=0} u_{0}$ is in $W_{2}^{1}(\Omega)$ and the function $\phi$ defined above is in $W_{2}^{1}(\Omega)^{3}$ and satisfies:

$$
\|\phi\|_{W_{2}^{1}(\Omega)} \leq C\left\|u_{0}\right\|_{W_{2}^{2}(\Omega)}^{2}
$$

Therefore System (5.7) recasts in

$$
\begin{aligned}
& \eta\left(\left.u_{t}\right|_{t=0}-\phi\right)+\left.\nabla P\right|_{t=0}=\nu \Delta u_{0}-\eta \phi \text { in } \Omega \\
& \operatorname{div}\left(\left.u_{t}\right|_{t=0}-\phi\right)=0 \text { in } \Omega \\
& \left.\left(\left.u_{t}\right|_{t=0}-\phi\right)\right|_{\partial \Omega}=0 \text { on } \partial \Omega .
\end{aligned}
$$

Now, testing the first equation by $\left(\left.u_{t}\right|_{t=0}-\phi\right)$ we get:

$$
\int_{\Omega} \eta\left|u_{t}\right|_{t=0}-\left.\phi\right|^{2} d x \leq \int_{\Omega} \eta^{-1}\left|\nu \Delta u_{0}-\eta \phi\right|^{2} d x
$$

Thus, due to (5.8), we discover that $\left.u_{t}\right|_{t=0}$ is in $L_{2}(\Omega)$ and that

$$
\left\|\left.u_{t}\right|_{t=0}\right\|_{L_{2}(\Omega)} \leq C_{\nu, \eta}\left(\left\|u_{0}\right\|_{W_{2}^{2}(\Omega)}+\left\|u_{0}\right\|_{W_{2}^{2}(\Omega)}^{2}\right) .
$$

At this point, we would like to apply an energy method to (5.6). However, as $\operatorname{div}_{u} u_{t}$ may be nonzero, one cannot eliminate the term coming from $P_{t}$ (which is out of control). So we modify $(5.6)_{2}$ by introducing a vector-field $\xi$ so that

$$
\begin{array}{lr}
\operatorname{div}_{u} \xi=-\operatorname{div}\left(A_{t} u\right) & \text { in } \Omega, \\
\xi=0 & \text { on } \partial \Omega .
\end{array}
$$

We need $\xi$ to satisfy suitable estimates (in terms of the right-hand side) in $L_{\infty}\left(0, T ; L_{2}(\Omega)\right) \cap$ $L_{2}\left(0, T ; W_{2}^{1}(\Omega)\right)$ and $\xi_{t}$ to be bounded in $L_{2}(\Omega \times(0, T))$. This may be done by means of a Bogovskil type operator construction as in [12]. Here we shall define $\xi$ (treating $t$ as parameter) according to Lemma 6 in the Appendix.

\footnotetext{
${ }^{3}$ In fact, the function $\phi$ is in $W_{2}^{2}(\Omega)$ but we shall not take advantage of this in what follows.
} 
Let us start with the bound in $L_{\infty}\left(0, T ; L_{2}(\Omega)\right)$ : we have

$$
\|\xi\|_{L_{\infty}\left(0, T ; L_{2}(\Omega)\right)} \lesssim\left\|A_{t} u\right\|_{L_{\infty}\left(0, T ; L_{2}(\Omega)\right)}
$$

Therefore, using the fact that

$$
A_{t}=\left(\sum_{k \geq 0}(k+1)(-1)^{k+1}\left(\int_{0}^{t} D_{y} u d \tau\right)^{k}\right) \cdot D_{y} u
$$

we get (remember (5.1))

$$
\begin{aligned}
\|\xi\|_{L_{\infty}\left(0, T ; L_{2}(\Omega)\right)} & \lesssim\|u \otimes \nabla u\|_{L_{\infty}\left(0, T ; L_{2}(\Omega)\right)} \\
& \lesssim\|u\|_{L_{\infty}\left(0, T ; L_{\infty}(\Omega)\right)}\|\nabla u\|_{L_{\infty}\left(0, T ; L_{2}(\Omega)\right)} .
\end{aligned}
$$

In order to bound the right-hand side of (5.13), we apply the following classical parabolic estimate (which is related to our definition of Besov spaces in (1.3)):

$$
\|u\|_{L_{\infty}\left(0, T ; B_{p, p}^{2-2 / p}(\Omega)\right)} \leq C\left(\left\|u_{0}\right\|_{B_{p, p}^{2-2 / p}(\Omega)}+\left\|u_{t}, \nabla^{2} u\right\|_{L_{p}(\Omega \times(0, T))}\right) .
$$

Now, owing to Sobolev embedding, it is clear that the left-hand side of (5.14) controls the $L_{\infty}$ norm whenever $2-2 / p>n / p$, that is $p>(n+2) / 2$. The constant in (5.14) is time independent. Therefore, for any $m \in\left((n+2) / 2, n^{*}\right)$, we have

$$
\begin{aligned}
\|u\|_{L_{\infty}(\Omega \times(0, T))} & \leq C_{m}\left(\left\|u_{0}\right\|_{B_{m, m}^{2-2 / m}(\Omega)}+\left\|u_{t}, \nabla^{2} u\right\|_{L_{m}(\Omega \times(0, T))}\right) \\
& \leq C_{m}\left(\left\|u_{0}\right\|_{B_{n^{*}, n^{*}}^{2-2 / n^{*}}(\Omega)}+T^{\frac{1}{m}-\frac{1}{n^{*}}} \Xi_{(u, P)}(T)\right) .
\end{aligned}
$$

Inequality (5.14) with $p=2$ also yields

$$
\begin{aligned}
\|\nabla u\|_{L_{\infty}\left(0, T ; L_{2}(\Omega)\right.} & \lesssim\left\|u_{0}\right\|_{W_{2}^{1}(\Omega)}+\left\|u_{t}, \nabla^{2} u\right\|_{L_{2}(\Omega \times(0, T))} \\
& \lesssim\left\|u_{0}\right\|_{W_{2}^{1}(\Omega)}+T^{\frac{1}{2}-\frac{1}{n^{*}}} \Xi_{(u, P)}(T) .
\end{aligned}
$$

So, putting (5.13), (5.15) and (5.16) together, we get for some $\delta>0$,

$$
\|\xi\|_{L_{\infty}\left(0, T ; L_{2}(\Omega)\right)} \lesssim\left\|u_{0}\right\|_{W_{2}^{2}(\Omega)}^{2}+T^{\delta} \Xi_{(u, P)}^{2}(T) .
$$

Next, in order to bound $\xi$ in $L_{2}\left(0, T ; W_{2}^{1}(\Omega)\right)$, we use the fact that

$$
\operatorname{div}_{u} \xi=-A_{t}: D u \text {. }
$$

In effect, owing to (3.13), one may write $\operatorname{div}\left(A u_{t}\right)=A: D u_{t}$, hence the above relation may be obtained by taking the time derivative of $\operatorname{div}(A u)=A: D u$. So, using Lemma 6 and remembering that $n^{*}>n$, we get, for some $\delta>0$,

$$
\begin{aligned}
\|\xi\|_{L_{2}\left(0, T ; W_{2}^{1}(\Omega)\right)} & \leq C\left\|\left.\nabla u\right|^{2}\right\|_{L_{2}(\Omega \times(0, T))} \\
& \leq C\|\nabla u\|_{L_{\infty}\left(0, T ; L_{2}(\Omega)\right)}\|\nabla u\|_{L_{2}\left(0, T ; L_{\infty}(\Omega)\right)} \\
& \leq C\left(\left\|\nabla u_{0}\right\|_{L_{2}(\Omega)}+T^{1 / 2}\left\|\nabla u_{t}\right\|_{L_{2}(\Omega \times(0, T))}\right)\|\nabla u\|_{L_{2}\left(0, T ; W_{n^{*}}^{1}(\Omega)\right)} \\
& \leq C T^{\delta} \Xi_{(u, P)}(T)\left(\left\|u_{0}\right\|_{W_{2}^{1}(\Omega)}+T^{\delta} \Xi_{(u, P)}(T)\right) .
\end{aligned}
$$


Finally, let us bound $\xi_{t}$ in $L_{2}(\Omega \times(0, T))$. For that, we apply the last part of Lemma 6 which yields

$$
\left\|\xi_{t}\right\|_{L_{2}(\Omega \times(0, T))} \leq C\left\|A_{t} \xi, A_{t t} u, A_{t} u_{t}\right\|_{L_{2}(\Omega \times(0, T))} .
$$

Using (5.13), (5.15) and (5.17), we get

$$
\begin{aligned}
&\left\|A_{t} \xi\right\|_{L_{2}(\Omega \times(0, T))} \leq C\|\nabla u\|_{L_{2}\left(0, T ; L_{\infty}(\Omega)\right)}\|\xi\|_{L_{\infty}\left(0, T ; L_{2}(\Omega)\right)} \\
& \leq C\left(\left\|u_{0}\right\|_{W_{2}^{2}(\Omega)}^{2}+T^{\delta} \Xi_{(u, P)}^{2}(T)\right)\|\nabla u\|_{L_{2}\left(0, T ; L_{\infty}(\Omega)\right)}, \\
&\left\|A_{t t} u\right\|_{L_{2}(\Omega \times(0, T))} \leq\left\|\nabla u_{t}\right\|_{L_{2}(\Omega \times(0, T))}\|u\|_{L_{\infty}\left(0, T ; L_{\infty}(\Omega)\right)} \\
& \leq C\left\|\nabla u_{t}\right\|_{L_{2}(\Omega \times(0, T))}\left(\left\|u_{0}\right\|_{W_{n^{*}}^{2-2 / n^{*}}(\Omega)}+T^{\delta} \Xi_{(u, P)}(T)\right), \\
&\left\|A_{t} u_{t}\right\|_{L_{2}(\Omega \times(0, T))} \leq C \| \nabla u \otimes u_{t} \|_{L_{2}(\Omega \times(0, T))} \\
& \leq C\|\nabla u\|_{L_{2}\left(0, T ; L_{\infty}(\Omega)\right)}\left(\left\|u_{t}-\xi\right\|_{L_{\infty}\left(0, T ; L_{2}(\Omega)\right)}+\|\xi\|_{L_{\infty}\left(0, T ; L_{2}(\Omega)\right)}\right) .
\end{aligned}
$$

So one may conclude that

$$
\begin{aligned}
\left\|\xi_{t}\right\|_{L_{2}(\Omega \times(0, T))} & \lesssim\left(\left\|u_{0}\right\|_{W_{2}^{2}(\Omega)}^{2}+T^{\delta} \Xi_{(u, P)}^{2}(T)\right)\|\nabla u\|_{L_{2}\left(0, T ; L_{\infty}(\Omega)\right)}+\left\|u_{0}\right\|_{W_{n^{*}}^{2-2 / n^{*}}(\Omega)} \\
& \left.+T^{\delta} \Xi_{(u, P)}(T)\right)\left\|\nabla u_{t}\right\|_{L_{2}\left(0, T ; L_{2}(\Omega)\right)}+\|\nabla u\|_{L_{2}\left(0, T ; L_{\infty}(\Omega)\right)}\left\|u_{t}-\xi\right\|_{L_{\infty}\left(0, T ; L_{2}(\Omega)\right)}
\end{aligned}
$$

Note that in (5.19), there is no factor $T^{\delta}$ for the leading order terms $\left\|u_{t}-\xi\right\|_{L_{\infty}\left(0, T ; L_{2}(\Omega)\right)}$ and $\left\|\nabla u_{t}\right\|_{L_{2}(\Omega \times(0, T))}$.

Once the vector-field $\xi$ has been constructed, one may recast System (5.6) in

$$
\begin{array}{ll}
\eta\left(u_{t}-\xi\right)_{t}-\nu \Delta\left(u_{t}-\xi\right)+\nabla_{u} P_{t} & \\
\quad=-\nu\left(\Delta-\Delta_{u}\right) u_{t}+\nu\left(\Delta_{u}\right)_{t} u-\left(\nabla_{u}\right)_{t} P-\eta \xi_{t}+\nu \Delta \xi & \text { in } \Omega \times(0, T), \\
\operatorname{div}_{u}\left(u_{t}-\xi\right)=0 & \text { in } \Omega \times(0, T), \\
u_{t}-\xi=0 & \text { on } \partial \Omega \times(0, T), \\
\left.\left(u_{t}-\xi\right)\right|_{t=0} \in L_{2}(\Omega) & \text { in }
\end{array}
$$

Note that, now, $\operatorname{div}_{u}\left(u_{t}-\xi\right)=0$ and that $\left.\left(u_{t}-\xi\right)\right|_{t=0}$ is in $L_{2}(\Omega)$ with

$$
\left\|\left.\left(u_{t}-\xi\right)\right|_{t=0}\right\|_{L_{2}(\Omega)} \leq C\left(\left\|u_{0}\right\|_{W_{2}^{2}(\Omega)}+\left\|u_{0}\right\|_{W_{2}^{2}(\Omega)}^{2}\right) .
$$

So taking the $L_{2}(\Omega)$-inner product of $(5.20)_{1}$ with $u_{t}-\xi$, there is no term generated by $\nabla_{u} P_{t}$ and we thus get

$$
\left.\frac{1}{2} \int_{\Omega} \eta\left|u_{t}-\xi\right|^{2} d x\right|_{t=T}+\nu \int_{0}^{T} \int_{\Omega}\left|\nabla\left(u_{t}-\xi\right)\right|^{2} d x d t \leq\left.\frac{1}{2} \int_{\Omega} \eta\left|u_{t}-\xi\right|^{2} d x\right|_{t=0}+\sum_{j=1}^{5} I_{j}
$$


with

$$
\begin{aligned}
& I_{1}:=C \nu \int_{0}^{T} \int_{\Omega}|(A-\mathrm{Id})|\left|\nabla u_{t}\right|\left|\nabla\left(u_{t}-\xi\right)\right| d x d t, \\
& I_{2}:=C \nu \int_{0}^{T} \int_{\Omega}|\nabla u|^{2}\left|\nabla\left(u_{t}-\xi\right)\right| d x d t, \\
& I_{3}:=C \int_{0}^{T} \int_{\Omega}|\nabla u||\nabla P|\left|u_{t}-\xi\right| d x d t, \\
& I_{4}:=\int_{0}^{T} \int_{\Omega} \eta\left|\xi_{t} \cdot\left(u_{t}-\xi\right)\right| d x d t, \\
& I_{5}:=\nu \int_{0}^{T} \int_{\Omega}\left|\nabla \xi \cdot \nabla\left(u_{t}-\xi\right)\right| d x d t .
\end{aligned}
$$

In order to bound terms $I_{1}, I_{2}, I_{3}$ and $I_{5}$, we use Hölder and Young inequalities. We get for all $\varepsilon>0$,

$$
\begin{aligned}
& I_{1} \leq \varepsilon \nu\left\|\nabla\left(u_{t}-\xi\right)\right\|_{L_{2}(\Omega \times(0, T))}^{2}+C_{\varepsilon, \nu}\|A-\operatorname{Id}\|_{L_{\infty}(\Omega \times(0, T))}^{2}\left\|\nabla u_{t}\right\|_{L_{2}(\Omega \times(0, T))}^{2}, \\
& I_{2} \leq \varepsilon \nu\left\|\nabla\left(u_{t}-\xi\right)\right\|_{L_{2}(\Omega \times(0, T))}^{2}+C_{\varepsilon, \nu}\left\||\nabla u|^{2}\right\|_{L_{2}(\Omega \times(0, T))}^{2}, \\
& I_{3} \leq \varepsilon\left\|u_{t}-\xi\right\|_{L_{\infty}\left(0, T ; L_{2}(\Omega)\right)}^{2}+C_{\varepsilon}\|\nabla u\|_{L_{2}\left(0, T ; L_{\infty}(\Omega)\right)}^{2}\|\nabla P\|_{L_{2}(\Omega \times(0, T))}^{2}, \\
& I_{5} \leq \varepsilon \nu\left\|\nabla\left(u_{t}-\xi\right)\right\|_{L_{2}(\Omega \times(0, T))}^{2}+C_{\varepsilon, \nu}\|\nabla \xi\|_{L_{2}(\Omega \times(0, T))}^{2} .
\end{aligned}
$$

Inequality (5.24) deserves a remark : in order to "close the estimates", we have to factor out the last term in the right-hand side by a quantity which is small enough when $T$ goes to 0 . Here this follows from the embedding $W_{n^{*}, n^{*}}^{2,1} \subset L_{2}\left(0, T ; L_{\infty}(\Omega)\right)$ which gives, because $n^{*}>2$,

$$
\|\nabla u\|_{L_{2}\left(0, T ; L_{\infty}(\Omega)\right)} \leq C T^{\frac{1}{2}-\frac{1}{n^{*}}\|u\|_{W_{n^{*}, n^{*}}^{2,1}(\Omega \times(0, T))}} .
$$

Finally, taking $m \in(1,2)$ so that $1=\frac{1}{m}+\frac{1}{n^{*}}$, and $\delta:=\frac{2}{m}-1$, we may write

$$
\begin{aligned}
I_{4} & \leq\left\|u_{t}-\xi\right\|_{L_{n^{*}(\Omega \times(0, T))}}\left\|\xi_{t}\right\|_{L_{m}(\Omega \times(0, T))}, \\
& \leq \varepsilon\left\|u_{t}-\xi\right\|_{L_{n^{*}(\Omega \times(0, T))}}^{2}+C_{\varepsilon} T^{\delta}\left\|\xi_{t}\right\|_{L_{2}(\Omega \times(0, T))}^{2} .
\end{aligned}
$$

Combining interpolation and Sobolev embedding, we may write for all $p \in(2, \infty)$,

$$
\left\|u_{t}-\xi\right\|_{L_{p}\left(0, T ; L_{q}(\Omega)\right)} \lesssim\left\|u_{t}-\xi\right\|_{L_{\infty}\left(0, T ; L_{2}(\Omega)\right)}^{1-2 / p}\left\|D\left(u_{t}-\xi\right)\right\|_{L_{2}(\Omega \times(0, T))}^{2 / p},
$$

with $n / q=n / 2-2 / p$. So taking $p=q=n^{*}:=2(n+2) / n$, we get

$$
\left\|u_{t}-\xi\right\|_{L_{n^{*}}(\Omega \times(0, T))} \leq C\left(\left\|u_{t}-\xi\right\|_{L_{\infty}\left(0, T ; L_{2}(\Omega)\right)}+\left\|\nabla\left(u_{t}-\xi\right)\right\|_{L_{2}(\Omega \times(0, T))}\right) .
$$

Therefore, the above estimates for $I_{1}$ to $I_{5}$ (with $\varepsilon$ small enough) eventually imply that

$$
\begin{aligned}
\left\|u_{t}-\xi\right\|_{L_{\infty}\left(0, T ; L_{2}(\Omega)\right)}^{2}+\nu\left\|\nabla\left(u_{t}-\xi\right)\right\|_{L_{2}(\Omega \times(0, T))}^{2} \leq 2\left\|\left.\left(u_{t}-\xi\right)\right|_{t=0}\right\|_{L_{2}(\Omega)}^{2} & \\
+ & C\left(\|\nabla u\|_{L_{2}\left(0, T ; L_{\infty}(\Omega)\right)}^{2}\left(\|\nabla u\|_{L_{\infty}\left(0, T ; L_{2}(\Omega)\right)}^{2}+\|\nabla P\|_{L_{2}(\Omega \times(0, T))}^{2}\right)\right. \\
& \left.+\|\nabla u\|_{L_{1}\left(0, T ; L_{\infty}(\Omega)\right)}^{2}\left\|\nabla u_{t}\right\|_{L_{2}(\Omega \times(0, T))}^{2}+T^{\delta}\left\|\xi_{t}\right\|_{L_{2}(\Omega \times(0, T))}^{2}+\|\nabla \xi\|_{L_{2}(\Omega \times(0, T))}^{2}\right),
\end{aligned}
$$


whence, using also the estimates for $\xi, \xi_{t}$ and for $\nabla u$ in $L_{2}\left(0, T ; L_{\infty}(\Omega)\right)$, we end up with

$$
\left\|u_{t}\right\|_{L_{\infty}\left(0, T ; L_{2}(\Omega)\right)}+\left\|\nabla u_{t}\right\|_{L_{2}(\Omega \times(0, T))} \leq C_{u_{0}}\left(1+T^{\delta} \Xi_{(u, P)}(T)\right)^{3} .
$$

Here $\delta>0$. Let us also stress that $C_{u_{0}}$ depends only on $\left\|u_{0}\right\|_{W_{2}^{2}(\Omega)},\left\|\rho_{0}\right\|_{L_{\infty}(\Omega)},\left\|\rho_{0}^{-1}\right\|_{L_{\infty}(\Omega)}$, $\Omega$ and $\nu$. In particular, it is time-independent.

Remark 5 At this stage, we find a limitation on the dimension of the domain: as we need to have $\nabla u \in L_{2}\left(0, T ; L_{\infty}(\Omega)\right)$, embedding requires that $n^{*}>n$. This is fulfilled if $n=2$ (because $2^{*}=4$ ) or $n=3$ (because $3^{*}=10 / 3$ ) but this is no longer satisfied in higher dimension. So we see that our method cannot be directly applied for $n \geq 4$ unless we differentiate the system with respect to time, more times. Physical motivation for considering dimension $n \geq 4$ is unclear, though.

Keeping (5.27) in mind, we see that in order to close the estimates, it suffices to bound the terms $\left\|\nabla^{2} u\right\|_{L_{n^{*}}(\Omega \times(0, T))}$ and $\|\nabla P\|_{L_{n^{*}}(\Omega \times(0, T))}$ which appear in the right-hand side of (5.28). For that, we rewrite System (5.4) as a stationary Stokes system, treating $\eta u_{t}$ as a source term, and the time variable as a parameter. So we consider

$$
\begin{array}{ll}
-\nu \Delta u+\nabla P=-\eta u_{t}-\nu\left(\Delta-\Delta_{u}\right) u+\left(\nabla-\nabla_{u}\right) P & \text { in } \Omega \times(0, T), \\
\operatorname{div} u=\operatorname{div}((\operatorname{Id}-A) u)=-A: D u & \text { in } \Omega \times(0, T), \\
u=0 & \text { on } \partial \Omega \times(0, T) .
\end{array}
$$

Note that one may use Proposition 3 so as to handle the potential part of $u$. Therefore using standard results for the stationary Stokes equation (see [15]) enables us to get

$$
\begin{aligned}
\left\|\nu \nabla^{2} u, \nabla P\right\|_{L_{n^{*}}(\Omega \times(0, T))} \leq & C\left(\left\|u_{t}\right\|_{L_{n^{*}}(\Omega \times(0, T))}\right. \\
& \left.+\left\|\nu\left(\Delta-\Delta_{u}\right) u,\left(\nabla-\nabla_{u}\right) P, \nu \nabla(A: D u)\right\|_{L_{n^{*}}(\Omega \times(0, T))}\right) .
\end{aligned}
$$

The key to bounding the right-hand side is that, because $n^{*}>n$, we have by embedding and Hölder's inequality

$$
\begin{aligned}
\left\|\int_{0}^{t} D u\left(t^{\prime}, y\right) d t^{\prime}\right\|_{L_{\infty}(\Omega \times(0, T))} & \leq C T^{1-\frac{1}{n^{*}}}\|\nabla u\|_{L_{n^{*}}\left(0, T ; L_{\infty}(\Omega)\right)} \\
& \leq C T^{1-\frac{1}{n^{*}}}\left\|\nabla^{2} u\right\|_{L_{n^{*}}(\Omega \times(0, T))} .
\end{aligned}
$$

In particular, this allows to write that

$$
\begin{aligned}
\left\|\left(\Delta-\Delta_{u}\right) u\right\|_{L_{n^{*}}(\Omega \times(0, T))} \lesssim & \left\|D\left(A^{T} A\right)\right\|_{L_{\infty}\left(0, T ; L_{n^{*}}(\Omega)\right.}\|D u\|_{L_{n^{*}}\left(0, T ; L_{\infty}(\Omega)\right.} \\
& +\left\|\operatorname{Id}-A^{T} A\right\|_{L_{\infty}(\Omega \times(0, T))}\left\|D^{2} u\right\|_{L_{n^{*}}(\Omega \times(0, T))}, \\
& \lesssim\left\|D^{2} u\right\|_{L_{1}\left(0, T ; L_{n^{*}}(\Omega)\right.}\|D u\|_{L_{n^{*}}\left(0, T ; L_{\infty}(\Omega)\right)} \\
& \quad+\|D u\|_{L_{1}\left(0, T ; L_{\infty}(\Omega)\right)}\left\|D^{2} u\right\|_{L_{n^{*}}(\Omega \times(0, T))}, \\
& \lesssim T^{1-1 / n^{*}} \Xi_{(u, P)}^{2}(T) .
\end{aligned}
$$

Similar estimates hold true for the other terms of the right-hand side of (5.30). So finally, putting together all the above inequalities leads to

$$
\Xi_{(u, P)}(T) \leq C_{u_{0}}\left(1+T^{\delta} \Xi_{(u, P)}(T)\right)^{3}
$$

for some $\delta=\delta(n) \in(0,1)$ which may be computed explicitly. 
Then we are able to close the estimate, namely to write that

$$
\Xi_{(u, P)}(T) \leq 8 C_{u_{0}}
$$

whenever $T$ has been chosen so that

$$
8 C_{u_{0}} T^{\delta} \leq 1
$$

\subsection{The proof of existence}

In this short subsection, we explain how the proof of existence may be achieved from the above a priori estimates.

Taking for granted the proof of the existence of a solution in the smooth case is the shortest way. Under the assumptions of Theorem 2, one may for instance smooth out the initial density $\rho_{0}$ by convolution by a positive mollifier. This provides us with a family of smooth approximate densities $\left(\rho_{0}^{\varepsilon}\right)_{\varepsilon>0}$ satisfying the same lower and upper bound as $\rho_{0}$. Then applying the local and existence and uniqueness statement of e.g. [6] (bounded domain case) or [8] (whole space case), one obtains a family of solutions $\left(\rho^{\varepsilon}, v^{\varepsilon}, \nabla Q^{\varepsilon}\right)$ for System 0.1 with data $\left(\rho_{0}^{\varepsilon}, v_{0}\right)$. This family of solutions has the required regularity. In addition, the possible blow-up of $\left(\rho^{\varepsilon}, v^{\varepsilon}, \nabla Q^{\varepsilon}\right)$ at time $T$ is controlled by the norm of $D v^{\varepsilon}$ in $L_{\infty}\left(0, T ; L_{2}(\Omega)\right) \cap L_{1}\left(0, T ; L_{\infty}(\Omega)\right)$. Note that Proposition 2 ensures that $\left(\rho^{\varepsilon}, v^{\varepsilon}, \nabla Q^{\varepsilon}\right)$ corresponds to a solution $\left(\eta^{\varepsilon}, u^{\varepsilon}, \nabla P^{\varepsilon}\right)$ of (3.10) with the same regularity.

Now, the computations that have been performed in the previous section, combined with the aforementioned blow-up criterion ensure that the lifespan of $\left(\eta^{\varepsilon}, u^{\varepsilon}, \nabla P^{\varepsilon}\right)$ (or of $\left(\rho^{\varepsilon}, v^{\varepsilon}, \nabla Q^{\varepsilon}\right)$ ) may be bounded by below as in (5.33), and that (5.32) is satisfied. The important point is that all those bounds depend on the density only through its infimum and supremum. So eventually, $\left(\rho^{\varepsilon}, v^{\varepsilon}, \nabla Q^{\varepsilon}\right)$ is uniformly bounded in

$$
L_{\infty}(\Omega \times(0, T)) \times W_{n^{*}, n^{*}}^{2,1}(\Omega \times(0, T)) \times L_{n^{*}}(\Omega \times(0, T)),
$$

and in addition, $\partial_{t} v^{\varepsilon}$ is bounded in $L_{\infty}\left(0, T ; L_{2}(\Omega)\right) \cap L_{2}\left(0, T ; W_{2}^{1}(\Omega)\right)$.

By resorting to standard compactness argument, it is now easy to conclude that this family converges, up to extraction, to some $(\rho, v, \nabla Q)$ with the same regularity and satisfying the same bounds. The regularity is so high that the it is clear that it satisfies (0.1). Uniqueness then follows from Theorem 1.

Remark 6 An alternative approach to the issue of existence can be done by an iterative scheme performed in the same way as in our recent work [11], or as in [27, 31] for the homogeneous Navier-Stokes equations in the Lagrangian coordinates.

\section{Global existence}

This section is dedicated to the proof of global-in-time solutions. As pointed out in the introduction, in the case of smooth data, this is a classical issue that has been solved by different authors in the Eulerian framework : if there is no vacuum initially then global 
existence may be achieved for general (smooth) data in the two-dimensional case and if the velocity is small in the three-dimensional case (see e.g. [8, 21]).

As for us, in order to show the global existence, one may adopt the Eulerian approach, too. However the very low regularity of the density will enforce us to treat the inhomogeneity of the fluid as a perturbation (hence to assume (1.7) or (1.9)) and to use the Lagrangian framework to prove the uniqueness.

\subsection{The two-dimensional case}

Here we prove Theorem 3. We concentrate on the proof of global a priori estimates. Indeed, existence can be established by an elementary approximation with smooth density exactly as in the previous section : for smooth enough densities, the existence of global solutions with velocity (locally) in $W_{4,2}^{2,1}\left(\Omega \times \mathbb{R}_{+}\right)$is ensured by [7] (bounded case) or by [8] (whole space case). In addition, let us emphasize that, in dimension two, this regularity guarantees that we are allowed to change coordinates between the Eulerian and Lagrangian ones. So the uniqueness follows from Theorem 1.

For getting the global existence, the computations are simpler in the Eulerian framework. We aim at getting a control over $v_{t}$ in $L_{\infty}\left(0, T ; L_{2}(\Omega)\right)$ in terms of the data and of $T$ only. Even though it is classical (see e.g. [3, 8, 21]) we here recall how to proceed. First, we test the momentum equation of System $(0.1)$ by $v_{t}$. We get:

$$
\int_{\Omega} \rho\left|v_{t}\right|^{2} d x+\frac{\nu}{2} \frac{d}{d t} \int_{\Omega}|\nabla v|^{2} d x+\int_{\Omega} \sqrt{\rho} v_{t} \cdot(\sqrt{\rho} v \cdot \nabla v) d x=0 .
$$

Hence Hölder and Young inequalities imply that

$$
\left\|\sqrt{\rho} v_{t}\right\|_{L_{2}(\Omega)}^{2}+\nu \frac{d}{d t}\|\nabla v\|_{L_{2}(\Omega)}^{2} \leq\|\sqrt{\rho} v\|_{L_{4}(\Omega)}^{2}\|\nabla v\|_{L_{4}(\Omega)}^{2} .
$$

On the other hand, using maximal regularity for the stationary Stokes equation

$$
\begin{array}{cl}
-\nu \Delta v+\nabla Q=\sqrt{\rho}\left(\sqrt{\rho} v_{t}+\sqrt{\rho} v \cdot \nabla v\right) & \text { in } \Omega \\
\operatorname{div} v=0 & \text { in } \Omega \\
v=0 & \text { on } \partial \Omega
\end{array}
$$

gives (omitting the time-dependency)

$$
\nu\left\|\nabla^{2} v\right\|_{L_{2}(\Omega)}+\|\nabla Q\|_{L_{2}(\Omega)} \lesssim\|\sqrt{\rho}\|_{L_{\infty}(\Omega)}\left(\left\|\sqrt{\rho} v_{t}\right\|_{L_{2}(\Omega)}+\|\sqrt{\rho} v\|_{L_{4}(\Omega)}\|\nabla v\|_{L_{4}(\Omega)}\right) .
$$

Now applying Ladyzhenskaya inequality $\|\nabla v\|_{L_{4}(\Omega)}^{2} \lesssim\|\nabla v\|_{L_{2}(\Omega)}\left\|\nabla^{2} v\right\|_{L_{2}(\Omega)}$, yields

$$
\begin{aligned}
\nu\left\|\nabla^{2} v\right\|_{L_{2}(\Omega)}+\|\nabla Q\|_{L_{2}(\Omega)} & \lesssim\|\sqrt{\rho}\|_{L_{\infty}(\Omega)}\left\|\sqrt{\rho} v_{t}\right\|_{L_{2}(\Omega)} \\
+ & \| \frac{\|\rho\|_{L_{\infty}(\Omega)}\|\sqrt{\rho} v\|_{L_{4}(\Omega)}^{2}\|\nabla v\|_{L_{2}(\Omega)}}{\nu} .
\end{aligned}
$$

Making use of Ladyzhenskaya inequality in (6.1), also leads to

$$
\left\|\sqrt{\rho} v_{t}\right\|_{L_{2}(\Omega)}^{2}+\nu \frac{d}{d t}\|\nabla v\|_{L_{2}(\Omega)}^{2} \leq \nu^{2} \frac{\left\|\nabla^{2} v\right\|_{L_{2}(\Omega)}^{2}}{\|\rho\|_{L_{\infty}(\Omega)}}+C \frac{\|\rho\|_{L_{\infty}(\Omega)}}{\nu^{2}}\|\sqrt{\rho} v\|_{L_{4}(\Omega)}^{4}\|\nabla v\|_{L_{2}(\Omega)}^{2} .
$$


Finally, adding up (6.4) and (6.3), using that $\|\rho(t)\|_{L_{\infty}(\Omega)}=\left\|\rho_{0}\right\|_{L_{\infty}(\Omega)}$ and performing a time integration yields

$$
\begin{aligned}
\|\nabla v(t)\|_{L_{2}(\Omega)}^{2}+\int_{0}^{t}\left(\frac{\left\|\sqrt{\rho} v_{t}\right\|_{L_{2}(\Omega)}^{2}}{2 \nu}\right. & \left.+\frac{\|\nabla Q\|_{L^{2}}^{2}}{\nu\left\|\rho_{0}\right\|_{L_{\infty}(\Omega)}}+\nu \frac{\left\|\nabla^{2} v\right\|_{L_{2}(\Omega)}^{2}}{\left\|\rho_{0}\right\|_{L_{\infty}(\Omega)}}\right) d \tau \\
& \leq\left\|\nabla v_{0}\right\|_{L_{2}(\Omega)}^{2}+\frac{C\left\|\rho_{0}\right\|_{L_{\infty}(\Omega)}}{\nu^{3}} \int_{0}^{t}\|\sqrt{\rho} v\|_{L_{4}(\Omega)}^{4}\|\nabla v\|_{L_{2}(\Omega)}^{2} d \tau,
\end{aligned}
$$

and Gronwall lemma implies that

$$
\begin{array}{r}
\nu\|\nabla v(t)\|_{L_{2}(\Omega)}^{2}+\int_{0}^{t}\left(\left\|\sqrt{\rho} v_{t}\right\|_{L_{2}(\Omega)}^{2}+\left\|\rho_{0}\right\|_{L_{\infty}(\Omega)}^{-1}\|\nabla Q\|_{L_{2}(\Omega)}^{2}+\nu^{2}\left\|\rho_{0}\right\|_{L_{\infty}(\Omega)}^{-1}\left\|\nabla^{2} v\right\|_{L_{2}(\Omega)}^{2}\right) d \tau \\
\leq \nu\left\|\nabla v_{0}\right\|_{L_{2}(\Omega)}^{2} e^{C \nu^{-3}\left\|\rho_{0}\right\|_{L_{\infty}(\Omega)} \int_{0}^{t}\|\sqrt{\rho} v\|_{L_{4}(\Omega)}^{4} d \tau} .
\end{array}
$$

Note that the exponential term is controlled thanks to the basic energy equality (1.1) (combined with Ladyzhenskaya's inequality).

Since $W_{2}^{1}\left(\mathbb{R}^{2}\right)$ is not embedded into $L_{\infty}\left(\mathbb{R}^{2}\right)$ we still do not control the change of coordinates so that we cannot apply Theorem 1 to get uniqueness. So we are required to improve the regularity of the solution to (0.1). In fact, it turns out to be possible to obtain $W_{q, p}^{2,1}$ smoothness for any $1<p<\infty$ and $n<q<\infty$ via bootstrap method. To avoid technicality, we focus on the case $p=2$ and $q=4$ which suffices both to perform the change of coordinates and to apply the uniqueness result stated in Theorem 1 . We rewrite System (0.1) as

$$
\begin{array}{llr}
m v_{t}-\nu \Delta v+\nabla Q=(m-\rho) v_{t}-\rho v \cdot \nabla v & \text { in } & \Omega \times(0, T), \\
\operatorname{div} v=0 & \text { in } \Omega \times(0, T), \\
v=0 & \text { in } \partial \Omega \times(0, T) .
\end{array}
$$

where $m=\inf _{y \in \Omega} \rho_{0}(y)$. Note that the method of characteristics ensures that the initial density controls lower and upper pointwise bounds of the density over $\Omega \times(0, T)$.

Then using Theorem 5 we get:

$$
\begin{aligned}
& \sup _{0 \leq t \leq T} \sqrt{m \nu}\|v(t)\|_{B_{4,2}^{1}(\Omega)}+\left\|m v_{t}, \nu \nabla^{2} v, \nabla Q\right\|_{L_{2}\left(0, T ; L_{4}(\Omega)\right)} \\
& \quad \leq C\left(\left\|(\rho-m) v_{t}\right\|_{L_{2}\left(0, T ; L_{4}(\Omega)\right)}+\|\rho v \cdot \nabla v\|_{L_{2}\left(0, T ; L_{4}(\Omega)\right)}+\sqrt{m \nu}\left\|v_{0}\right\|_{B_{4,2}^{1}(\Omega)}\right)
\end{aligned}
$$

Now, we have

$$
\|\rho v \cdot \nabla v\|_{L_{2}\left(0, T ; L_{4}(\Omega)\right)} \leq C\|v\|_{L_{4}\left(0, T ; L_{\infty}(\Omega)\right)}\|\nabla v\|_{L_{4}(\Omega \times(0, T))} .
$$

The right-hand side of (6.7) is bounded by means of (6.5) as

$$
W_{2,2}^{2,1} \subset L_{4}\left(0, T ; L_{\infty}(\Omega) \cap W_{4}^{1}(\Omega)\right) .
$$

The first term of the right-hand side of (6.7) can be absorbed by the left-hand side provided $c$ is sufficiently small in (1.7). This enables us to justify that the velocity $v$ remains in $W_{4,2}^{2,1}(\Omega \times(0, T))$ for all $T>0$.

Finally, as $4 \geq 2=\operatorname{dim} \Omega$, we are allowed to apply Theorem 1 in order to get the uniqueness of our constructed solutions. Theorem 3 is proved. 


\subsection{Global existence in the $n$-dimensional case}

In this part we address the global solvability issue in bounded $n$-dimensional domains with $n \geq 3$. We adopt the Lagrangian framework (however the Eulerian framework may be used as well, as regards the existence theory). The result is based on the technique for the homogeneous system performed in [26].

In contrast with the other sections, working in bounded domains is important: this is due to the following result which ensures the exponential decay of the energy norm.

Lemma 4 Let $u$ be a sufficiently smooth solution to (3.10). Then

$$
\frac{1}{2} \frac{d}{d t} \int_{\Omega} \eta|u|^{2} d y+\nu \int_{\Omega}\left|\nabla_{u} u\right|^{2} d y=0
$$

as long as the Lagrangian coordinates are defined. In addition if $\Omega$ is bounded then

$$
\|u(t)\|_{L_{2}(\Omega)}^{2} \leq e^{-\frac{\nu \lambda_{1}}{\eta^{*}} t}\left\|u_{0}\right\|_{L_{2}(\Omega)}^{2} .
$$

where $\lambda_{1}$ stands for the first eigenvalue of the Laplace operator, and $\eta^{*}=\|\eta\|_{L_{\infty}(\Omega)}$.

Proof: The proof is similar to that of Lemma 1 : testing $(3.10)_{2}$ by $u$ we get (6.9).

In order to get (6.10), it suffices to notice that, owing to incompressibility, we have

$$
\int_{\Omega} \eta|u|^{2} d y=\int_{\Omega} \rho|v|^{2} d x \quad \text { and } \quad \int_{\Omega}\left|\nabla_{u} u\right|^{2} d y=\int_{\Omega}|\nabla v|^{2} d x
$$

Hence using (1.1) and Poincaré's inequality, we readily get (6.10).

\section{Proof of Theorem 4:}

We focus on the proof of global a priori estimates for smooth solutions to (3.10). Indeed, from those estimates, it is easy to proceed as in Section 5 so as to prove the existence of a global solution under the assumptions of Theorem 4: this is only a matter of smoothing out the initial density so as to construct a sequence of smooth solutions (given by e.g. [7]) with uniform norms.

So given a global solution $(\eta, u, \nabla P)$ to (3.10) with data $\rho_{0} \in L_{\infty}(\Omega)$ satisfying (1.9) and $u_{0} \in B_{q, p}^{2-2 / p}(\Omega)$ with $\operatorname{div} u_{0}=0$ and $\left.u_{0}\right|_{\partial \Omega}=0$, we introduce the following quantities:

$$
\begin{gathered}
M_{-1}:=m^{1 / p} \nu^{1 / p^{\prime}}\left\|u_{0}\right\|_{B_{q, p}^{2-2 / p}(\Omega)}+m\left\|u_{0}\right\|_{L_{2}(\Omega)}, \\
M_{k}:=m^{1 / p} \nu^{1 / p^{\prime}}\|u\|_{L_{\infty}\left(k, k+1 ; B_{q, p}^{2-2 / p}(\Omega)\right)}+\left\|m u_{t}, \nu \nabla^{2} u, \nabla P\right\|_{L_{p}\left(k, k+1 ; L_{q}(\Omega)\right)}
\end{gathered}
$$

where $m:=\inf \rho_{0}$ and $k \in \mathbb{N}$. Recall $1<p<\infty, n<q<\infty$.

Let us notice that setting

$$
u(t, x)=\nu \widetilde{u}(\nu t, x) \quad \text { and } \quad P(t, x)=\nu^{2} \widetilde{P}(\nu t, x)
$$

reduces our study to the case $\nu=1$. Hence we shall assume from now on that $\nu=1$. 
Define a smooth function $\zeta: \mathbb{R} \rightarrow[0,1]$ such that

$$
\zeta^{k}(t)= \begin{cases}1 & \text { if } t \geq 0 \\ 0 & \text { if } t \leq-1\end{cases}
$$

and set $\zeta^{k}(t):=\zeta(t-k)$ for $k \geq 0$, and $I_{k}:=[k-1, k+1]$ for $k \geq 1$.

We recast System (3.10) with $t_{0}=k-1$ as follows:

$$
\begin{aligned}
& m\left[\zeta^{k} u\right]_{t}-\Delta\left[\zeta^{k} u\right]+\nabla\left[\zeta^{k} P\right]=\zeta^{k}(m-\eta) u_{t} \\
& -\left(\Delta-\Delta_{u}\right)\left[\zeta^{k} u\right]+\left(\nabla-\nabla_{u}\right)\left[\zeta^{k} P\right]+m\left(\zeta^{k}\right)_{t} u \quad \text { in } \quad \Omega \times(0, T), \\
& \operatorname{div}\left[\zeta^{k} u\right]=\operatorname{div}\left[\zeta^{k} u\right]-\operatorname{div}_{u}\left[\zeta^{k} u\right] \quad \text { in } \Omega \times(0, T) \text {, } \\
& \zeta^{k} u=0 \quad \text { on } \partial \Omega \times(0, T) \text {, } \\
& \left.\zeta^{k} u\right|_{t=k-1}=0 \quad \text { in } \Omega \text {. }
\end{aligned}
$$

Let $m^{*}:=\sup \rho_{0}$. We claim that there exist two positive constants $K$ and $\alpha$ depending only on $m^{*}, n, \Omega, p, q$, so that, under Condition (1.9), we have

$$
M_{k} \leq K M_{-1} e^{-\alpha k} \quad \text { for all } k \in \mathbb{N} .
$$

Let us observe that, by Sobolev embedding (here we use that $q>n$ ), we have

$$
\int_{0}^{k+1}\|D u\|_{L_{\infty}(\Omega)} d s \leq C \sum_{\ell=0}^{k}\left\|D^{2} u\right\|_{L_{p}\left(I_{\ell} ; L_{q}(\Omega)\right)} \leq C \sum_{\ell=0}^{k} M_{k} .
$$

So, given that

$$
\sum_{k \geq 0} K M_{-1} e^{-\alpha k}=\frac{K M_{-1}}{1-e^{-\alpha}}
$$

if we assume that $M_{-1}$ is small enough -a condition which is equivalent to the smallness of $c^{\prime}$ in (1.9)- then (5.1) is satisfied on $[0, k+1]$ if (6.13) is satisfied up to $k$.

Proving (6.13) will be done by induction on $k$. The first step, $k=0$, is clear. This is a direct consequence of Theorem 5 applied to (3.10) on the time interval $[0,1]$, and of estimates for $A$.

Let us now take for granted Inequality (6.13) up to $k-1$. In order to prove it for $k$, we shall estimate $\left(\zeta^{k} u, \zeta^{k} P\right)$ on the interval $I_{k}$. For that, one may resort once again to Theorem 5. First, we bound the right-hand side of $(6.12)$ in $L_{p}\left(I_{k} ; L_{q}(\Omega)\right)$ : we readily have

$$
\begin{aligned}
\left\|\zeta^{k}(m-\eta) u_{t}\right\|_{L_{p}\left(I_{k} ; L_{q}(\Omega)\right)} \leq & \left(m^{*}-m\right)\left\|u_{t}\right\|_{L_{p}\left(I_{k} ; L_{q}(\Omega)\right)}, \\
\left\|\left(\Delta-\Delta_{u}\right)\left[\zeta^{k} u\right]\right\|_{L_{p}\left(I_{k} ; L_{q}(\Omega)\right)} \leq & \left\|A^{T} A-\operatorname{Id}\right\|_{L_{\infty}\left(\Omega \times I_{k}\right)}\left\|\nabla^{2} u\right\|_{L_{p}\left(I_{k} ; L_{q}(\Omega)\right)} \\
& \quad+\left\|\nabla\left(A^{T} A\right)\right\|_{L_{\infty}\left(I_{k} ; L_{q}(\Omega)\right)}\|\nabla u\|_{L_{p}\left(I_{k} ; L_{\infty}(\Omega)\right)}, \\
\left\|\left(\nabla-\nabla_{u}\right)\left[\zeta^{k} P\right]\right\|_{L_{p}\left(I_{k} ; L_{q}(\Omega)\right)} \leq & \|A-\operatorname{Id}\|_{L_{\infty}\left(\Omega \times I_{k}\right)}\|\nabla P\|_{L_{p}\left(I_{k} ; L_{q}(\Omega)\right)}, \\
\left\|m\left(\zeta^{k}\right)_{t} u\right\|_{L_{p}\left(I_{k} ; L_{q}(\Omega)\right)} & \leq m^{*}\|u\|_{L_{p}\left(I_{k} ; L_{q}(\Omega)\right)} .
\end{aligned}
$$

Let us notice that, by interpolation and because $\Omega$ is bounded, we have for some $\theta \in(0,1)$,

$$
\|u\|_{L_{p}\left(I_{k} ; L_{q}(\Omega)\right)} \leq C\left\|D^{2} u\right\|_{L_{p}\left(I_{k} ; L_{q}(\Omega)\right)}^{\theta}\|u\|_{L_{p}\left(I_{k} ; L_{2}(\Omega)\right)}^{1-\theta} .
$$


Therefore, taking advantage of (6.10) and of the definition of $M_{k-1}$ and of $M_{k}$, we get for some $\beta>0$ (depending only on $\Omega, p, q, m_{*}$ and for all $\varepsilon \in(0,1)$,

$$
m^{*}\|u\|_{L_{p}\left(I_{k} ; L_{q}(\Omega)\right)} \leq \varepsilon\left(M_{k-1}+M_{k}\right)+C_{\varepsilon} M_{-1} e^{-\beta k} .
$$

Next, we have to bound the left-hand side of $(6.12)_{2}$ : we have

$$
\begin{aligned}
&\left\|\operatorname{div}\left((\operatorname{Id}-A)\left[\zeta^{k} u\right]\right)\right\|_{L_{p}\left(I_{k} ; W_{q}^{1}(\Omega)\right)} \leq\|A-\operatorname{Id}\|_{L_{\infty}\left(\Omega \times I_{k}\right)}\left\|\nabla^{2} u\right\|_{L_{p}\left(I_{k} ; L_{q}(\Omega)\right)} \\
&+\|\nabla A\|_{L_{\infty}\left(I_{k} ; L_{q}(\Omega)\right)}\|\nabla u\|_{L_{p}\left(I_{k} ; L_{\infty}(\Omega)\right)} \\
&\left\|\partial_{t}\left((\operatorname{Id}-A) \zeta^{k} u\right)\right\|_{L_{p}\left(I_{k} ; L_{q}(\Omega)\right)} \leq\|A-\operatorname{Id}\|_{L_{\infty}(\Omega \times(0, T))}\left\|\left(\zeta^{k} u\right)_{t}\right\|_{L_{p}\left(I_{k} ; L_{q}(\Omega)\right)} \\
&+\left\|A_{t}\right\|_{L_{p}\left(I_{k} ; L_{\infty}(\Omega)\right)}\|u\|_{L_{\infty}\left(I_{k} ; L_{q}(\Omega)\right)} .
\end{aligned}
$$

Let us look at the quantities depending on the matrix $A$. Recall that

$$
A^{-1}=\mathrm{Id}+\int_{0}^{t} D u(s) d s,
$$

so taking advantage of (6.14) and of the hypothesis that follows, one may write that

$$
\|\operatorname{Id}-A\|_{L_{\infty}(\Omega \times(0, k+1))} \leq 2\|D u\|_{L_{1}\left(0, k+1 ; L_{\infty}(\Omega)\right)},
$$

and a similar inequality for $\mathrm{Id}-A^{T} A$. Likewise, we have

$$
\|D A\|_{L_{\infty}\left(I_{k} ; L_{q}(\Omega)\right)} \leq C \sum_{\ell=0}^{k}\left\|D^{2} u\right\|_{L_{q}\left(I_{\ell} ; L_{q}(\Omega)\right)} \leq C \sum_{\ell=0}^{k} M_{\ell}
$$

and

$$
\left\|A_{t}\right\|_{L_{p}\left(I_{k} ; L_{\infty}(\Omega)\right)} \leq C\|D u\|_{L_{p}\left(I_{k} ; L_{\infty}(\Omega)\right.} \leq C\left(M_{k-1}+M_{k}\right) .
$$

So finally, putting together all the previous inequalities and applying Theorem 5 to (6.12), we end up with

$$
\begin{aligned}
& \| m\left(\zeta^{k} u_{t}\right), \nabla^{2}\left(\zeta^{k} u\right), \nabla\left(\zeta^{k} P\right) \\
& \quad \leq C\left(M_{k-1}+M_{k}\right)\left(\left(\frac{m^{*}-m}{m}\right)+\left(\sum_{\ell=0}^{k} M_{\ell}\right)+\varepsilon\right)+C_{\varepsilon} M_{-1} e^{-\beta k} .
\end{aligned}
$$

At this point, it is clear that one has to take $\alpha=\beta$. Note also that if $M_{-1}$ and the oscillations of the density are small enough then, taking $\varepsilon$ small enough too, the above inequality implies, up to a change of $C$,

$$
M_{k} \leq C\left(c M_{k-1}+M_{-1} e^{-\alpha k}\right)
$$

Now, using the induction hypothesis (6.13) for $M_{k-1}$, we deduce that

$$
M_{k} \leq K M_{-1} e^{-\alpha k}\left(\frac{c C e^{\alpha}}{K}+\frac{C}{K}\right) .
$$

Therefore, we see that if we take $K=2 C$ and assume that $c$ has been chosen so that $c C e^{\alpha} \leq 1 / 2$ then we get (6.13) for $M_{k}$. 
Note that our proof is not quite rigorous as we did use (6.13) at rank $k$ in the above inequalities. To make the argument work, it is just a matter of replacing the interval $I_{k}$ with $[k-1, T]$. By continuity of the norms with respect to time, it is clear that (6.13) at rang $\ell \leq k-1$ ensures that the desired inequality is satisfied on $[k, T]$ for any $T$ close enough to $k$. Then resorting to a standard bootstrap argument allows to conclude to the desired inequality for $M_{k}$. This completes the proof of Theorem 4.

\section{Appendix}

Throughout this paper, we used repeatedly the following well-known result for the divergence equation (see e.g. [15] and the references therein):

Lemma 5 Let $\Omega$ be a bounded Lipschitz domain of $\mathbb{R}^{n}$. There exists a linear operator $\mathcal{B}$ which is bounded from $L_{q}(\Omega)$ to $W_{q}^{1}(\Omega)$ for all $q \in(1, \infty)$ and such that for any $f \in L_{q}(\Omega)$ the vector-field $u:=\mathcal{B}(f)$ satisfies

$$
\operatorname{div} u=f \quad \text { in } \Omega \quad \text { and }\left.\quad u\right|_{\partial \Omega}=0 \quad \text { on } \partial \Omega .
$$

This result may be proved by means of an explicit formula - the Bogovskir formula - that provides a solution to the above divergence equation in the case where $\Omega$ is star-shaped. In our paper, we had to use a more elaborate version of the above lemma, namely the following statement that has been established in [12]:

Proposition 3 Let $\Omega$ be a $C^{2}$ bounded domain. There exists a linear operator $B$ acting on couples $(R, \zeta)$ with $R: \Omega \rightarrow \mathbb{R}^{n}$ and $\zeta: \partial \Omega \rightarrow \mathbb{R}$ which is continuous from $L_{q}\left(\Omega ; \mathbb{R}^{n}\right) \times$ $W_{q}^{-1 / q}(\partial \Omega, \mathbb{R})$ to $L_{q}\left(\Omega, \mathbb{R}^{n}\right)$ for all $q \in(1,+\infty)$ and such that $u:=B(R, \zeta)$ satisfies the generalized divergence equation:

$$
-\int_{\Omega} u \cdot \nabla \phi d x=-\int_{\Omega} R \cdot \nabla \phi d x+\int_{\partial \Omega} \zeta \phi d \sigma \text { for all } \phi \in C^{\infty}(\bar{\Omega}) .
$$

If in addition $\operatorname{div} R \in L_{q}(\Omega)$ and $R \cdot \vec{n}=0$ then $u:=B(R, 0)$ satisfies

$$
\operatorname{div} u=\operatorname{div} R \quad \text { in } \Omega \quad \text { and }\left.\quad u\right|_{\partial \Omega}=0 \quad \text { on } \partial \Omega
$$

and the following inequality holds true:

$$
\|u\|_{W_{q}^{1}(\Omega)} \leq C\|\operatorname{div} R\|_{L_{q}(\Omega)} .
$$

Furthermore, if we also have $\operatorname{div} R \in W_{q}^{1}(\Omega)$ then $u$ is in $W_{q}^{2}(\Omega)$ and we have

$$
\|u\|_{W_{q}^{2}(\Omega)} \leq C\|\operatorname{div} R\|_{W_{q}^{1}(\Omega)} .
$$

We claim that this statement implies Lemma 2. Indeed, we set $u:=B(R, 0)$. Then it is is clear that (2.4) holds true. Then differentiating $u$ with respect to time yields

$$
u_{t}=B\left(R_{t}, 0\right)
$$

Hence applying the first part of the above statement yields (2.5). 
In Section 5, owing to our use of Lagrangian coordinates, it was natural to extend Lemma 2 and Proposition 3 to the twisted divergence equation, namely

$$
\operatorname{div}_{A} u=\operatorname{div} R \text { in } \Omega \quad \text { and } u=0 \text { on } \partial \Omega
$$

with $\operatorname{div}_{A} u:=\operatorname{div}(A u)$. In particular, we used the following statement:

Lemma 6 Let $\Omega$ be a $C^{2}$ bounded domain. Let $A \in L_{\infty}\left(\Omega ; \mathbb{R}^{n \times n}\right)$ be such that $\operatorname{det} A \equiv 1$. There exists a positive constant $c$ such that if

$$
\|A-\operatorname{Id}\|_{L_{\infty}(\Omega)} \leq c
$$

then there exists a map $B_{A}$ acting on couples $(R, \zeta)$ with $R: \Omega \rightarrow \mathbb{R}^{n}$ and $\zeta: \partial \Omega \rightarrow \mathbb{R}$ which is continuous from $L_{q}\left(\Omega ; \mathbb{R}^{n}\right) \times W_{q}^{-1 / q}(\partial \Omega, \mathbb{R})$ to $L_{q}\left(\Omega, \mathbb{R}^{n}\right)$ for all $q \in(1,+\infty)$ and such that $u:=B_{A}(R, \zeta)$ satisfies the generalized twisted divergence equation:

$$
-\int_{\Omega} A u \cdot \nabla \phi d x=-\int_{\Omega} R \cdot \nabla \phi d x+\int_{\partial \Omega} \zeta \phi d \sigma \text { for all } \phi \in C^{\infty}(\bar{\Omega}) .
$$

If in addition $\operatorname{div} R \in L_{q}(\Omega)$ and $R \cdot \vec{n}=0$ then $u:=B_{A}(R, 0)$ satisfies

$$
\operatorname{div}_{A} u=A: D u=\operatorname{div} R \text { in } \Omega \quad \text { and } u=0 \text { on } \partial \Omega \text {, }
$$

and (7.3) with a constant independent of $A$.

Finally, in the smooth case, if the data $R$ and $A$ depend on a parameter $t$ in some interval of $\mathbb{R}$ with $R_{t}$ in $L_{q}(\Omega)$ and (7.5) satisfied for almost all $t$, then $u$ fulfills:

$$
\left\|u_{t}\right\|_{L_{q}(\Omega)} \leq C\left(\left\|A_{t} u\right\|_{L_{q}(\Omega)}+\left\|R_{t}\right\|_{L_{q}(\Omega)}\right) .
$$

Proof: The proof follows from Proposition 3: we consider the linear operator $T$ defined by

$$
T(\bar{\xi})=\xi, \text { where } \xi:=B((\operatorname{Id}-A) \bar{\xi}+R, \zeta) .
$$

Note that this definition and the fact that $\operatorname{det} A \equiv 1$ imply that any fixed point of $T$ satisfies (7.6) (or (7.7) in the smooth case with $\zeta=0$ ). Next, under Condition (7.5) with $c$ small enough, one may apply the Banach fixed point theorem to $T$ so as to get a solution to our problem. The reader may refer to [12] for more details.

Concerning the proof of inequality (7.8), it suffices to differentiate once the equality

$$
u=B((\operatorname{Id}-A) u+R, 0)
$$

We get

$$
u_{t}=B\left(-A_{t} u+(\mathrm{Id}-A) u_{t}+R_{t}, 0\right) .
$$

So it is a mere consequence of the first part of Proposition 3.

Acknowledgement. The second author (PBM) thanks Vladimir Šverak for a fruitful discussion. The second author has been supported by the MN grant IdP2011 000661. 


\section{References}

[1] H. Abels: The initial-value problem for the Navier-Stokes equations with a free surface in $L^{q}$-Sobolev spaces, Adv. Differential Equations, 10(1), pages 45-64 (2005).

[2] F. Ammar Khodja and M.M. Santos: 2D density-dependent Leray problem with a discontinuous density, Methods Appl. Anal., 13(4), pages 321-335 (2006).

[3] S. Antontsev, A. Kazhikhov and V. Monakhov: Boundary value problems in mechanics of nonhomogeneous fluids. Studies in Mathematics and its Applications, 22. NorthHolland Publishing Co., Amsterdam, 1990.

[4] A. J. Archer: Dynamical density functional theory for molecular and colloidal fluids: A microscopic approach to fluid mechanics, J. Chem. Phys. 130, 014509 ; doi:10.1063/1.3054633 (8 pages) (2009)

[5] O. V. Besov, V. P. I'lin and S. M. Nikolskij: Integral Function Representation and Imbedding Theorem, Moscow, 1975.

[6] Y. Cho and H. Kim: Unique solvability for the density-dependent Navier-Stokes equations, Nonlinear Analysis, 59(4), pages 465-489 (2004).

[7] R. Danchin: Density-dependent incompressible fluids in bounded domains, Journal of Mathematical Fluid Mechanics, 8, pages 333-381 (2006).

[8] R. Danchin, Local and global well-posedness results for flows of inhomogeneous viscous fluids. Adv. Differential Equations, 9 (2004), no. 3-4, 353-386.

[9] R. Danchin: Density-dependent incompressible viscous fluids in critical spaces, Proceedings of the Royal Society of Edinburgh, Sect. A, 133(6), pages 1311-1334 (2003).

[10] R. Danchin and P.B. Mucha: The divergence equation in rough spaces, Journal of Mathematical Analysis and Applications, 386, pages 10-31 (2012).

[11] R. Danchin and P.B. Mucha: A Lagrangian approach for the incompressible NavierStokes equations with variable density, to appear in Communications on Pure and Applied Mathematics.

[12] R. Danchin and P.B. Mucha: Divergence, submitted.

[13] B. Desjardins: Regularity Results for Two-Dimensional Flows of Multiphase Viscous Fluids, Arch. Rational Mech. Anal., 137, pages 135-158 (1997).

[14] E. DiBenedetto, Degenerate parabolic equations. Springer-Verlag, New York, 1993

[15] G. Galdi: An introduction to the mathematical theory of the Navier-Stokes equations. Vol. I. Linearized steady problems. Springer Tracts in Natural Philosophy, 38, SpringerVerlag, New York, 1994. 
[16] P. Germain: Strong solutions and weak-strong uniqueness for the nonhomogeneous Navier-Stokes equation, J. Anal. Math., 105, pages 169-196 (2008).

[17] Y. Giga abd H. Sohr: Abstract $L^{p}$ estimates for the Cauchy problem with applications to the Navier-Stokes equations in exterior domains, J. Funct. Anal., 102, pages 72-94 (1991).

[18] D. Hoff: Uniqueness of weak solutions of the Navier-Stokes equations of multidimensional compressible flow, SIAM Journal on Mathematical Analysis, 37(6), pages 1742-1760 (2006).

[19] D.N. Ku: Blood flow in arteries, Annual Review of Fluid Mechanics, 29, pages 399-434 (1997).

[20] P.-L. Lions: Mathematical Topics in Fluid Dynamics, Vol. 1 Incompressible Models, Oxford University Press (1996).

[21] O. Ladyzhenskaya and V. Solonnikov: The unique solvability of an initial-boundary value problem for viscous incompressible inhomogeneous fluids, Journal of Soviet Mathematics, 9, pages 697-749 (1978).

[22] O. A. Ladyzhenskaya, V. A. Solonnikov and N. N. Ural'ceva: Linear and Quasilinear Equations of Parabolic Type, R.I. Amer. Math. Soc., Providence (1968).

[23] Y. Liu, L. Zhang, X. Wang and W.K. Liu: Coupling of Navier-Stokes equations with protein molecular dynamics and its application to hemodynamics, International Journal for Numerical Methods in Fluids, Special Issue: International Conference on Finite Element Methods in Fluids Volume 46, Issue 12, pages 1237-1252, (2004).

[24] P. Maremonti and V.A. Solonnikov: On nonstationary Stokes problem in exterior domains, Ann. Scuola Norm. Sup. Pisa Cl. Sci., 24(3), pages 395-449 (1997).

[25] P.B. Mucha: On an estimate for the linearized compressible Navier-Stokes equations in the $L^{p}$-framework, Colloq. Math., 87(2), pages 159-169 (2001).

[26] P.B. Mucha: Stability of nontrivial solutions of the Navier-Stokes system on the three dimensional torus, J. Differential Equations, 172(2), pages 359-375 (2001).

[27] P.B. Mucha and W.M. Zajączkowski: On local existence of solutions of free boundary problem for incompressible viscous self-gravitating fluid motion, Applicationes Mathematicae, 27(3), pages 319-333 (2000).

[28] P.B. Mucha and W.M. Zajączkowski: On a $L^{p}$-estimate for the linearized compressible Navier-Stokes equations with the Dirichlet boundary conditions. J. Differential Equations, 186(2), pages 377-393 (2002).

[29] M.M. Santos: Stationary solution of the Navier-Stokes equations in a 2D bounded domain for incompressible flow with discontinuous density. Z. Angew. Math. Phys., 53(4), pages 661-675 (2002). 
[30] Y. Shibata and S. Shimizu: On a resolvent estimate of the Stokes system in a half space arising from a free boundary problem for the Stokes equations, Math. Nachr., 282, pages 482-499 (2009).

[31] V.A. Solonnikov: On the nonstationary motion of isolated value of viscous incompressible fluid, Izv. AN SSSR, 51(5), pages 1065-1087 (1987).

[32] I. Tice and Y. Wang : The viscous surface-internal wave problem: nonlinear RayleighTaylor instability, arXiv:1109.5657v1 (2011).

[33] H. Triebel: Interpolation theory, function spaces, differential operators. North-Holland Mathematical Library, 18. , Amsterdam-New York, 1978. 